WZ Scattering at the LHC

\title{
in the Littlest Higgs Model
}

by

\section{Kenneth P. Moats}

\author{
A thesis submitted to the \\ Faculty of Graduate Studies and Research \\ in partial fulfillment of the requirements \\ for the degree of \\ Master of Science \\ Department of Physics \\ Carleton University \\ Ottawa-Carleton Institute of Physics \\ Ottawa, Canada
}

September 12, 2007

Copyright (C) 2007 Kenneth P. Moats 


$\begin{array}{ll}\begin{array}{l}\text { Library and } \\ \text { Archives Canada }\end{array} & \begin{array}{l}\text { Bibliothèque et } \\ \text { Archives Canada }\end{array} \\ \begin{array}{l}\text { Published Heritage } \\ \text { Branch }\end{array} & \begin{array}{l}\text { Direction du } \\ \text { Patrimoine de l'édition }\end{array} \\ \begin{array}{l}\text { 395 Wellington Street } \\ \text { Ottawa ON K1A 0N4 }\end{array} & \begin{array}{l}\text { 395, rue Wellington } \\ \text { Ottawa ON K1A ON4 } \\ \text { Canada }\end{array} \\ \end{array}$

Your file Votre référence ISBN: 978-0-494-33707-3 Our file Notre référence ISBN: 978-0-494-33707-3

NOTICE:

The author has granted a nonexclusive license allowing Library and Archives Canada to reproduce, publish, archive, preserve, conserve, communicate to the public by telecommunication or on the Internet, loan, distribute and sell theses worldwide, for commercial or noncommercial purposes, in microform, paper, electronic and/or any other formats.

The author retains copyright ownership and moral rights in this thesis. Neither the thesis nor substantial extracts from it may be printed or otherwise reproduced without the author's permission.
AVIS:

L'auteur a accordé une licence non exclusive permettant à la Bibliothèque et Archives Canada de reproduire, publier, archiver, sauvegarder, conserver, transmettre au public par télécommunication ou par l'Internet, prêter, distribuer et vendre des thèses partout dans le monde, à des fins commerciales ou autres, sur support microforme, papier, électronique et/ou autres formats.

L'auteur conserve la propriété du droit d'auteur et des droits moraux qui protège cette thèse. $\mathrm{Ni}$ la thèse ni des extraits substantiels de celle-ci ne doivent être imprimés ou autrement reproduits sans son autorisation.
In compliance with the Canadian

Privacy Act some supporting forms may have been removed from this thesis.

While these forms may be included in the document page count, their removal does not represent any loss of content from the thesis.
Conformément à la loi canadienne sur la protection de la vie privée, quelques formulaires secondaires ont été enlevés de cette thèse.

Bien que ces formulaires aient inclus dans la pagination, il n'y aura aucun contenu manquant. 


\section{Abstract}

Little Higgs Models have recently been proposed as a mechanism for Electroweak Symmetry Breaking. A particular feature of the Littlest Higgs model is a triplet of heavy Higgs bosons, the singly-charged member of which can be produced at the Large Hadron Collider (LHC) through scattering of $W$ and $Z$ bosons. The calculation of the signal cross section for this process is carried out using the Effective Vector Boson Approximation and the Goldstone Equivalence Theorem. The relevant backgrounds are then calculated using the WHIZARD event generator. We find that the signal can be observed above the background for a triplet vacuum expectation value of $v^{\prime} / v=0.2$, but not for a more theoretically favoured value of $v^{\prime} / v=0.05$. The effect of double tagging the quark jets is also investigated and it is found that tagging a single energetic jet is more efficient in retaining the signal events. 


\section{Acknowledgements}

I am most indebted to my advisor, Stephen Godfrey, for agreeing to take me on as his student. His mentoring and patience are greatly appreciated, and his enthusiasm for research in particle physics has surely helped strengthen my interest in the subject.

I thank the other members of the Carleton phenomenology group, Pat Kalyniak, Heather Logan and Hong-Sheng Hou, for helpful comments and discussions. I am also grateful to Juergen Reuter for his help in learning to use the WHIZARD event generator. Fortunately, the Carleton Physics Department has a number of experimentalists working with the ATLAS detector at the LHC. In particular, I would like to thank fellow students J.P. Archambault and Malachi Schram for helping me understand some of the more experimental details related to the work in this thesis. I would also like to thank my fellow theory student Travis Martin, with whom I have worked rather closely over the past two years.

I thank my undergraduate physics professors at the University of Saskatchewan. I am particularly grateful to Rainer Dick and Tom Steele, for sparking my interest in particle physics. Without their guidance, I surely would not have decided to pursue a career in the field.

Most importantly, I would like to thank my family for their unending love and support during both graduate school as well as throughout my life. I am especially grateful to my parents, Murray and Brenda, for instilling in me the importance of education and for their endless supply of encouragement in all of my endeavors. 
Finally, I would like to dedicate this thesis to my brother, Brian William David Moats, who passed away during the course of this work. I will always treasure the time I was able to spend with him, and he continues to be very dearly missed. 


\section{Contents}

$\begin{array}{ll}\text { Abstract } & \text { iii }\end{array}$

Acknowledgements $\quad$ iv

Table of Contents $\quad$ vi

List of Tables vii

List of Figures viii

1 Introduction 1

1.1 The Standard Model . . . . . . . . . . . . . . . . . . 3

1.2 The Higgs Mechanism . . . . . . . . . . . . . . 5

1.3 Limits on the Higgs Boson Mass . . . . . . . . . . . . . . 11

1.4 Motivation for Physics Beyond the Standard Model . . . . . . . . . . 14

1.5 The Littlest Higgs Model . . . . . . . . . . . . . . . . . . 17

2 The WZ Scattering Signal 22

2.1 Longitudinally Polarized Vector Bosons . . . . . . . . . . . . . . . 24

2.2 The Effective Vector Boson Approximation . . . . . . . . . . . . 27

2.3 Parton Level Cross Section for $W_{L} Z_{L}$ Scattering . . . . . . . . . . 32

2.4 The Goldstone Equivalence Theorem . . . . . . . . . . . . . . . 34

vi 
3 Backgrounds to WZ Scattering $\quad 39$

3.1 Description of Backgrounds . . . . . . . . . . . . . 40

3.2 The WHIZARD Event Generator . . . . . . . . . . . . . . . 42

3.3 Kinematics of Final State Particles . . . . . . . . . . . . . 44

4 Results $\quad 55$

5 Conclusions $\quad 61$

A Feynman Rules $\quad 63$

vii 


\section{List of Tables}

3.1 Leptonic cuts and jet cuts used in $W Z$ scattering to enhance the signal to background ratio. Note that the central jet veto cuts are to be interpreted as: "Reject all events with jets having $|y|<2.0$ and $p_{T}>$ $100 \mathrm{GeV} . \ldots \ldots \ldots \ldots$

A.1 Feynman rules required to calculate the parton level amplitude for $W Z$ scattering in the Littlest Higgs model. All particles are assumed to be outgoing. . . . . . . . . . . . . . . .

A.2 Feynman rules required to calculate the parton level amplitude for $W_{L} Z_{L}$ scattering in the Littlest Higgs model using the Goldstone Equivalence Theorem. All particles are assumed to be outgoing. . . . 


\section{List of Figures}

1.1 Theoretical limits on the Higgs boson mass. The upper bound follows from triviality conditions and the lower bound follows from the requirement that $V(v)<V(0)$. Also shown is the upper limit permitted by precision electroweak measurements at the $95 \%$ confidence level. The range of allowed Higgs masses shown by the white region predicts a light Higgs boson [14]. . . . . . . . . . . . . . . . . . . 15

2.1 $W Z$ scattering at the LHC. The incoming quarks from the colliding protons emit $W$ and $Z$ bosons, which interact with each other. The $W$ and $Z$ bosons emerge in the final state, along with two spectator

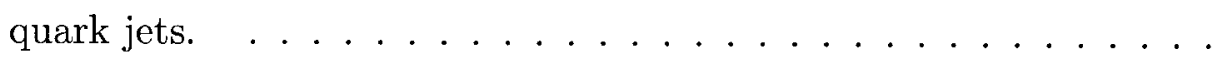

2.2 The CTEQ6M parton distribution functions at $\mathrm{Q}=2$ and $100 \mathrm{GeV}$. .

2.3 Feynman diagrams for $W Z$ scattering. In the Standard Model, only diagrams (a) - (d) are present. In the Littlest Higgs model, diagrams (e) - (g) must also be taken into account. . . . . . . . . . . . . 
2.4 Feynman diagrams for $W_{L} Z_{L}$ scattering in the Littlest Higgs model using the Goldstone Equivalence Theorem. The external longitudinal gauge bosons of Figure 2.3 have been replaced by the corresponding unphysical Goldstone bosons. . . . . . . . . . . . . . . 36

2.5 Invariant mass distribution for $W_{L} Z_{L}$ scattering. The dotted curve shows the result for $W_{L} Z_{L}$ scattering in the Standard Model and the other two curves are for $W_{L} Z_{L}$ scattering in the Littlest Higgs model. The solid curve was obtained using longitudinally polarized gauge bosons and the dashed curve was obtained using the Goldstone Equivalence Theorem. These two curves agree quite well, especially at

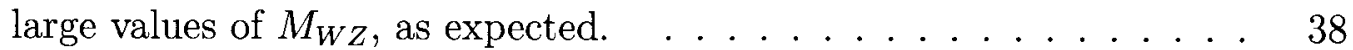

3.1 The electroweak background to $W_{L} Z_{L}$ scattering, in which at least one of the vector bosons is transversely polarized. The electroweak background also includes diagrams in which $W$ and $Z$ bosons are radiated by the incoming quarks via electroweak interactions. Note that this irreducible background contains spectator quark jets in the final state. 41

3.2 The di-boson production background. This background does not involve any quark jets and can be suppressed by requiring a forward jet

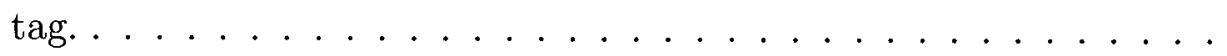


3.3 Backgrounds from heavy quark processes, arising from $t \bar{t}$ production followed by $t$ and $\bar{t}$ decays to real $W$ and $Z$ bosons. The jets in this background process tend to be more central than those of the signal, so this background can be suppressed by imposing a central jet veto.

3.4 Transverse mass distributions of the backgrounds to $W Z$ scattering before imposing cuts for an integrated luminosity of $\mathcal{L}=100 \mathrm{fb}^{-1}$. .

3.5 Rapidity distributions for the three charged leptons. The signal events are shown as the solid line, and the electroweak background events are shown as the dashed line. . . . . . . . . . . . . . . . . . . . .

3.6 Transverse momentum distributions for the three charged leptons and for the missing transverse momentum. The signal events are shown as the solid line, and the electroweak background events are shown as the dashed line. . . . . . . . . . . . . . . . . . .

3.7 Transverse momentum distributions for the $\mathrm{Z}$ boson. The signal events are shown as the solid line, and the electroweak background events are shown as the dashed line. . . . . . . . . . . . . . . .

3.8 Rapidity distributions for the final state quark jets. The signal events are shown as the solid line, and the electroweak background events are shown as the dashed line. . . . . . . . . . . . . . . . . . .

3.9 Transverse momentum distributions for the final state quark jets. The signal events are shown as the solid line, and the electroweak background events are shown as the dashed line. . . . . . . . . . . 
3.10 Energy distributions for the final state quark jets. The signal events are shown as the solid line, and the electroweak background events are shown as the dashed line. . . . . . . . . . . . . . . .

4.1 Transverse mass distribution for the $W Z$ signal in the Littlest Higgs model, together with the background remnant after the cuts of Table 3.1, for an integrated luminosity of $\mathcal{L}=100 \mathrm{fb}^{-1}$. This plot was generated using WHIZARD with a Standard Model Higgs mass of $M_{H}=120 \mathrm{GeV}$, a heavy Higgs triplet mass of $M_{\Phi}=1.5 \mathrm{TeV}$, and a triplet vacuum expectation value of $v^{\prime} / v=0.2 \ldots \ldots \ldots \ldots$

4.2 Transverse mass distribution for the $W Z$ signal in the Littlest Higgs model, together with the background remnant after the cuts of Table 3.1, for an integrated luminosity of $\mathcal{L}=100 \mathrm{fb}^{-1}$. This plot was generated using WHIZARD with a Standard Model Higgs mass of $M_{H}=120 \mathrm{GeV}$, a heavy Higgs triplet mass of $M_{\Phi}=2.0 \mathrm{TeV}$, and a triplet vacuum expectation value of $v^{\prime} / v=0.2 \ldots \ldots \ldots \ldots$

4.3 Transverse mass distribution for the $W Z$ signal in the Littlest Higgs model, together with the background remnant after the cuts of Table 3.1 , for an integrated luminosity of $\mathcal{L}=100 \mathrm{fb}^{-1}$. This plot was generated using WHIZARD with a Standard Model Higgs mass of $M_{H}=120 \mathrm{GeV}$, a heavy Higgs triplet mass of $M_{\Phi}=1.0 \mathrm{TeV}$, and a triplet vacuum expectation value of $v^{\prime} / v=0.2 \ldots \ldots \ldots \ldots$. . . 59 
4.4 Transverse mass distribution for the $W Z$ signal in the Littlest Higgs model, together with the background remnant after the cuts of Table 3.1 , for an integrated luminosity of $\mathcal{L}=100 \mathrm{fb}^{-1}$. This plot was generated using WHIZARD with a Standard Model Higgs mass of $M_{H}=120 \mathrm{GeV}$, a heavy Higgs triplet mass of $M_{\Phi}=1.5 \mathrm{TeV}$, and a triplet vacuum expectation value of $v^{\prime} / v=0.2$. Double forward jet tagging was used in this plot. . . . . . . . . . . . . 60 


\section{Chapter 1}

\section{Introduction}

Our current knowledge of particle physics is described by the Standard Model, a theory that governs the interactions between fundamental particles. The Standard Model predicts the results of most experiments with impressive accuracy. However, despite the success of the Standard Model, the general consensus among particle physicists is that the Standard Model is unsatisfactory, and that it is only part of a more fundamental theory. One of the reasons for this concern is related to Electroweak Symmetry Breaking (EWSB), the mechanism by which particles acquire mass. In the Standard Model, EWSB occurs by the Higgs mechanism, which predicts the existence of a particle called the Higgs boson, which has not yet been discovered. However, there are theoretical inconsistencies in the Higgs Mechanism which lead us to believe that there must exist some type of new physics beyond the Standard Model.

Consequently, there has been much interest in the study of particle physics beyond the Standard Model, in which new theoretical models have been proposed in 
order to offer a more self-consistent description of EWSB. Although a considerable number of models have been proposed, only experimental data will reveal which one, if any, is correct. This comparison of theoretical predictions and experimental results is the foundation of an area of particle physics known as phenomenology. The year 2007 marks an exciting time for the particle physics community. Within the next year, experiments at the Large Hadron Collider (LHC) will begin taking data in order to study the mechanism for EWSB, using energies inaccessible at previous experiments. The long term goal of this work is to compare the predictions of EWSB in various models so that when data from the LHC become available, these models can be tested experimentally.

In this chapter, the Standard Model will be introduced, with emphasis on the electroweak sector of the theory. We give an overview of the Higgs mechanism and discuss the motivation for considering models of physics beyond the Standard Model. We then describe a recent model that has received much attention, the Littlest Higgs Model, which predicts the existence of several new particles at the $\mathrm{TeV}$ scale. In particular, we focus on the production of the singly charged heavy Higgs boson $\Phi^{+}$, which occurs via $W Z$ scattering. In Chapter 2, we discuss the $W Z$ scattering signal, and employ the Effective Vector Boson Approximation and the Goldstone Equivalence Theorem in order to simplify our calculations. One must also consider background processes in order to determine whether this signal can be experimentally observed at the LHC. This is the focus of Chapter 3. Finally, the results of this analysis are presented in Chapter 4, and we conclude with a discussion of these results in Chapter 5 . 


\subsection{The Standard Model}

According to our current knowledge of physics, there are four fundamental interactions that exist in nature: gravitation, electromagnetism, the weak interaction, and the strong interaction. Gravitation, described classically by the general theory of relativity (a satisfactory theory of quantum gravity has not yet been formulated), is the weakest of the four interactions and is assumed to play a negligible role in elementary particle physics. The electromagnetic interaction is best described by the theory of Quantum Electrodynamics (QED) and proceeds by the exchange of massless spin-one photons between charged particles. Weak interactions (first discovered in $\beta$ decay) are mediated by the massive spin-one $W^{ \pm}$and $Z^{0}$ bosons. Following the work of Glashow, Weinberg and Salam [1-3], the theories for the electromagnetic and weak interactions can be described by a single theory of electroweak interactions. The strong interaction, described by the theory of Quantum Chromodynamics (QCD), describes the interactions between quarks, which proceed by the exchange of massless spin-one gluons $[4,5]$.

The Standard Model (SM) of particle physics is a theory that encompasses the electromagnetic, weak and strong interactions. Particles in the Standard Model can be classified into two groups based on their spin: fermions (i.e. quarks and leptons) have half-integer spin, and bosons (i.e. $\gamma, W^{ \pm}, Z^{0}$ and the gluons) have integer spin. The SM group structure is $S U(3)_{c} \otimes S U(2)_{L} \otimes U(1)_{Y}$, where $c$ refers to the colour charge of QCD, $L$ refers to a left-handed group structure, and $Y$ is the hypercharge group. An observer in an inertial reference frame says that a particle is left-handed (right-handed) if its spin is oriented opposite (along) its direction of 
motion, as measured from that particular frame. Fermions (quarks and leptons) occur in left-handed doublets, along with right-handed singlets.

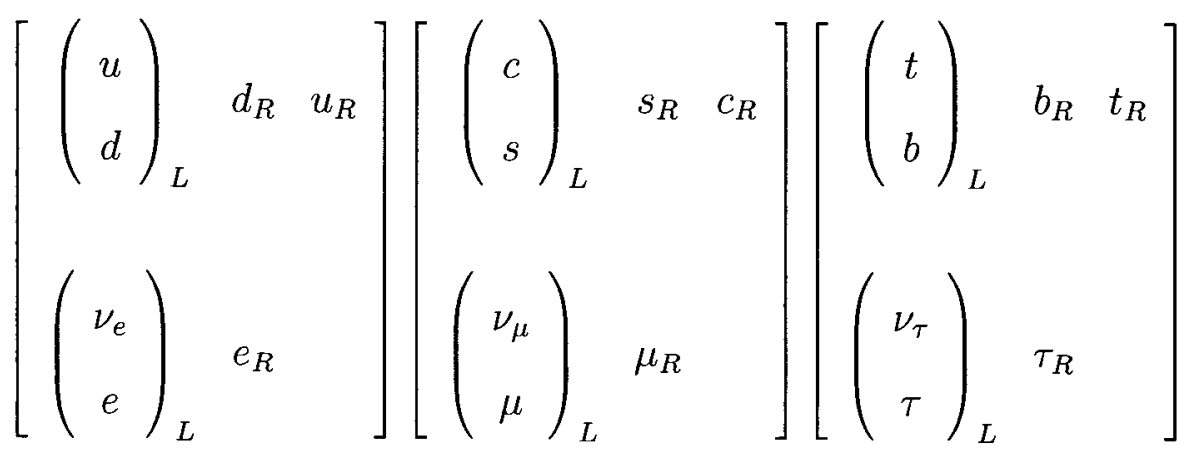

While all quarks and leptons transform non-trivially under $U(1)_{Y}$, only the lefthanded doublets transform non-trivially under $S U(2)_{L}$, and only the quarks and gluons carry the colour charge of QCD. One should note that although all the particles of these three generations have been observed experimentally, only the first generation is found in normal matter. Particles of the second and third generations are more massive and unstable, decaying to particles of the first generation.

In this thesis, we are interested primarily in the $S U(2)_{L} \otimes U(1)_{Y}$ theory of electroweak interactions, which predicts the existence of an additional spin-zero (scalar) particle called the Higgs boson. The interactions of the Standard Model particles with the Higgs boson are believed to be responsible for generating their masses. This process is called the Higgs mechanism and will be described in greater detail in the following section. 


\subsection{The Higgs Mechanism}

In this section, we introduce the Higgs mechanism of the Standard Model, following the notation of Ref. [6]. Consider the Glashow-Weinberg-Salam theory of electroweak interactions. It is an $S U(2)_{L} \otimes U(1)_{Y}$ gauge theory containing three $S U(2)_{L}$ gauge bosons, $W_{\mu}^{i}(i=1,2,3)$, with coupling constant $g$, and one $U(1)_{Y}$ gauge boson $B_{\mu}$ with coupling constant $g^{\prime}$. The kinetic energy terms of the electroweak Lagrangian are given by

$$
\mathcal{L}_{K E}=-\frac{1}{4} W_{\mu \nu}^{i} W^{\mu \nu i}-\frac{1}{4} B_{\mu \nu} B^{\mu \nu}
$$

where

$$
\begin{aligned}
B_{\mu \nu} & =\partial_{\mu} B_{\nu}-\partial_{\nu} B_{\mu} \\
W_{\mu \nu}^{i} & =\partial_{\mu} W_{\nu}^{i}-\partial_{\nu} W_{\mu}^{i}+g \epsilon^{i j k} W_{\mu}^{j} W_{\nu}^{k}
\end{aligned}
$$

This Lagrangian is required to be invariant under $U(1)$ and $S U(2)$ gauge transformations of the fields, given by

$$
\begin{aligned}
B_{\mu} & \rightarrow B_{\mu}+\partial_{\mu} \alpha(x) \\
W_{\mu}^{i} & \rightarrow W_{\mu}^{i}+\partial_{\mu} \Lambda^{i}(x)+g \epsilon^{i j k} \Lambda^{j}(x) W_{\mu}^{k} .
\end{aligned}
$$

If mass terms of the form $\mathcal{L}_{M_{B}} \sim M_{B}^{2} B_{\mu} B^{\mu}$ and $\mathcal{L}_{M_{W}} \sim M_{W}^{2} W_{\mu}^{i} W^{\mu i}$ are added to the Lagrangian, then it is no longer gauge invariant. This suggests that all four gauge bosons must be massless. However, in the electroweak theory, the photon is the only 
massless gauge boson; the current experimental values for the $W^{ \pm}$and $Z^{0}$ boson masses are $M_{W}=80.403 \pm 0.029 \mathrm{GeV}$ and $M_{Z}=91.1876 \pm 0.0021 \mathrm{GeV}$ [7]. The masses of the $W^{ \pm}$and $Z^{0}$ bosons are generated by a mechanism known as Electroweak Symmetry Breaking (EWSB), which, in the Standard Model, proceeds by the Higgs mechanism as described below.

The gauge fields are coupled to a complex scalar $S U(2)_{L}$ doublet

$$
\Phi=\left(\begin{array}{c}
\phi^{+} \\
\phi^{0}
\end{array}\right)
$$

as follows. Adding gauge invariant terms for the interaction and propagation of scalars, the Lagrangian of equation (1.2) becomes

$$
\mathcal{L}=\left(D^{\mu} \Phi\right)^{\dagger}\left(D_{\mu} \Phi\right)-V(\Phi)-\frac{1}{4} W_{\mu \nu}^{i} W^{\mu \nu i}-\frac{1}{4} B_{\mu \nu} B^{\mu \nu},
$$

where the covariant derivative of the scalar field, $D_{\mu} \Phi$, is given by

$$
D_{\mu} \Phi=\left(\partial_{\mu}+i \frac{g}{2} \tau \cdot W_{\mu}+i \frac{g^{\prime}}{2} B_{\mu}\right) \Phi
$$

and the scalar potential, $V(\Phi)$, is given by

$$
V(\Phi)=\mu^{2}\left|\Phi^{\dagger} \Phi\right|+\lambda\left|\Phi^{\dagger} \Phi\right|^{2}, \lambda>0 .
$$

If $\mu^{2}<0$, then $V(\Phi)$ is such that the state of minimum energy is not at $\Phi=0$, as would be the case for $\mu^{2}>0$. By convention, we choose the vacuum expectation 
value (vev) of $\Phi$ to correspond to the state of minimum energy, given by

$$
\langle\Phi\rangle=\frac{1}{\sqrt{2}}\left(\begin{array}{l}
0 \\
v
\end{array}\right)
$$

where $v=\sqrt{-\mu^{2} / \lambda}$.

In order to see the physical particle spectrum, the fields must be shifted about their vev as follows:

$$
\Phi=\frac{1}{\sqrt{2}} e^{i \frac{G \cdot \tau}{2 v}}\left(\begin{array}{c}
0 \\
v+H
\end{array}\right)
$$

where $G^{i}(i=1,2,3)$ and $H$ are four real fields with no vev, and the $\tau^{i}$ are the $2 \times 2$ Pauli matrices. Making a gauge transformation $\Phi \rightarrow e^{-i \frac{G \cdot \tau}{2 v}} \Phi$, results in the unitary gauge (where only the physical fields are present),

$$
\Phi=\frac{1}{\sqrt{2}}\left(\begin{array}{c}
0 \\
v+H
\end{array}\right) .
$$

The Lagrangian of equation (1.8) is clearly invariant under the transformation $\Phi \rightarrow$ $-\Phi$. However, if equation (1.13) is inserted into this Lagrangian, we find that it is not invariant under $H \rightarrow-H$. Therefore, we say that the electroweak symmetry is spontaneously broken. In fact, the symmetry breaking scheme is of the desired form: $S U(2)_{L} \otimes U(1)_{Y} \rightarrow U(1)_{E M}$. The $U(1)_{E M}$ symmetry remains unbroken, and electromagnetism is therefore mediated by a massless photon.

Substitution of equation (1.13) into the Lagrangian of equation (1.8) and retaining terms up to second order in the fields (i.e. kinetic energy or mass terms) leads 
to a scalar field, $H$, with mass

$$
M_{H}^{2}=2 v^{2} \lambda
$$

The physical gauge fields, $W^{ \pm}, Z$ and $\gamma$, are given by

$$
\begin{aligned}
W_{\mu}^{ \pm} & =\frac{1}{\sqrt{2}}\left(W_{\mu}^{1} \mp i W_{\mu}^{2}\right) \\
Z_{\mu} & =-s_{W} B_{\mu}+c_{W} W_{\mu}^{3} \\
A_{\mu} & =c_{W} B_{\mu}+s_{W} W_{\mu}^{3},
\end{aligned}
$$

where

$$
\begin{aligned}
& s_{W} \equiv \sin \theta_{W}=\frac{g^{\prime}}{\sqrt{g^{2}+g^{\prime 2}}} \\
& c_{W} \equiv \cos \theta_{W}=\frac{g}{\sqrt{g^{2}+g^{\prime 2}}} .
\end{aligned}
$$

Here, $\theta_{W}$ is the weak mixing angle and its experimental value is given by $\sin ^{2} \theta_{W}=$ $0.23122 \pm 0.00015[7]$. This also leads to a relation for the electromagnetic charge, $e$ :

$$
e=g \sin \theta_{W}=g^{\prime} \cos \theta_{W}
$$

After the symmetry breaking, the gauge bosons obtain masses given by

$$
\begin{aligned}
M_{W}^{2} & =\frac{1}{4} g^{2} v^{2} \\
M_{Z}^{2} & =\frac{1}{4}\left(g^{2}+g^{2}\right) v^{2} \\
M_{A} & =0 .
\end{aligned}
$$


The three unphysical fields, $G^{i}$, are called Goldstone bosons and through the above gauge transformation, we say that they have been "eaten" to give the $W^{ \pm}$and $Z$ bosons their mass. Consequently, the $W^{ \pm}$and $Z$ have acquired longitudinal polarization components. The photon, on the other hand, remains massless since the $U(1)_{E M}$ symmetry is unbroken, and consequently, does not acquire a longitudinal polarization. The physical scalar field, $H$, which remains in the theory is called the Higgs boson, and this process is known as the Higgs mechanism.

The Higgs mechanism also generates gauge invariant fermion masses as follows. Fermions can be written in terms of their left- and right-handed projections, $\psi=$ $\psi_{L}+\psi_{R}$, where

$$
\psi_{L, R}=\frac{1}{2}\left(1 \mp \gamma_{5}\right) \psi
$$

and

$$
\gamma_{5}=\left(\begin{array}{ll}
0 & 1 \\
1 & 0
\end{array}\right)
$$

Since left- and right-handed fermions transform differently under the chiral $S U(2)_{L} \otimes$ $U(1)_{Y}$ gauge groups, a fermion mass term of the form

$$
\mathcal{L}_{m_{f}}=-m_{f} \bar{\psi} \psi=-m_{f}\left(\bar{\psi}_{L} \psi_{R}+\bar{\psi}_{R} \psi_{L}\right)
$$

is not gauge invariant and therefore forbidden. However, a Higgs doublet, $\Phi$, with a vacuum expectation value, $v$, generates a gauge invariant mass term of the form

$$
\mathcal{L}_{m_{f}}=-\sqrt{2} \frac{m_{f}}{v}\left(\bar{\psi}_{L} \Phi \psi_{R}+\bar{\psi}_{R} \Phi^{\dagger} \psi_{L}\right)
$$


Experimentally, we know that the $W$ boson does not couple to right-handed fields, so $\psi_{R}$ is an $S U(2)_{L}$ singlet, whereas $\psi_{L}$ is an $S U(2)_{L}$ doublet. Notice that since the neutrino has no right-handed partner in the Standard Model, it remains massless according to equation (1.22).

The parameter $v$ can be found from the charged current for muon decay, $\mu \rightarrow e \bar{\nu}_{e} \nu_{\mu}$. Since the momentum carried by the $W$ boson is of the order $m_{\mu}$, it can be neglected compared to $M_{W}$, and this process can be considered as an effective interaction of four fermions following the work of Fermi [8]. This yields

$$
\frac{G_{F}}{\sqrt{2}}=\frac{g^{2}}{8 M_{W}^{2}}=\frac{1}{2 v^{2}}
$$

where $G_{F}=(1.16637 \pm 0.00001) \times 10^{-5} \mathrm{GeV}^{-2}$ is the interaction strength for muon decay [7]. This gives the result

$$
v=\left(\sqrt{2} G_{F}\right)^{-\frac{1}{2}}=246 \mathrm{GeV} .
$$

Now, since $v^{2}=-\frac{\mu^{2}}{\lambda}=(246 \mathrm{GeV})^{2}$ and $M_{H}^{2}=2 v^{2} \lambda$, the free parameters $\mu$ and $\lambda$ can be expressed in terms of $M_{H}$. Therefore, Higgs production and decay processes can be computed in terms of the Higgs mass alone. The Higgs boson mass given by equation (1.14) is a free parameter and, unfortunately, the value of $M_{H}$ is unknown since the Higgs boson has not yet been discovered. However, there are theoretical arguments that place limits on the value of $M_{H}$ and these are discussed in the next section. 


\subsection{Limits on the Higgs Boson Mass}

In order to facilitate experimental searches for the Higgs boson, it is useful to first consider theoretical limits on its mass. An upper bound on the Higgs boson mass can be obtained from triviality arguments as follows [9]. Consider a pure scalar theory in which the potential is given by equation (1.10), where the quartic coupling is $\lambda=M_{H}^{2} / 2 v^{2}$. Due to self interactions with the scalar field, the quartic coupling changes with the effective energy, $Q$, according to

$$
\frac{d \lambda}{d t}=\frac{3 \lambda^{2}}{4 \pi^{2}}
$$

where $t=\ln \left(Q^{2} / Q_{0}^{2}\right)$ and $Q_{0}$ is some reference scale (often chosen to be $v$ in the Standard Model). The solution of equation (1.25) is

$$
\frac{1}{\lambda(Q)}=\frac{1}{\lambda\left(Q_{0}\right)}-\frac{3}{4 \pi^{2}} \ln \left(\frac{Q^{2}}{Q_{0}^{2}}\right)
$$

If the Standard Model is to be valid for arbitrarily large energies $(\lambda(Q)$ remains finite as $Q \rightarrow \infty$ ), then $\lambda\left(Q_{0}\right) \rightarrow 0$, making it a non-interacting (trivial) theory at low energies. To obtain a bound on the Higgs mass requires that the quartic coupling is never negative, which is an unphysical value:

$$
\frac{1}{\lambda(\Lambda)}>0
$$

where $\Lambda$ is some large scale where new physics enters. Taking $Q_{0}=v$ in equation (1.26), and using the condition of equation (1.27), gives an upper bound on the Higgs 
boson mass

$$
M_{H}^{2}<\frac{8 \pi^{2} v^{2}}{3 \ln \left(\Lambda^{2} / v^{2}\right)}
$$

Requiring that there are no new physics before the Planck scale, $\Lambda=10^{19} \mathrm{GeV}$, yields

$$
M_{H}<144 \mathrm{GeV}
$$

Notice that this bound on $M_{H}$ becomes weaker for smaller values of $\Lambda$; if $\Lambda \sim 3 T e V$, the bound is roughly $M_{H}<600 \mathrm{GeV}$.

The above estimates were obtained for a theory involving only scalars. If the couplings to fermions and gauge bosons are included in the theory, the physics involved is different. Since the top quark couples most strongly to the Higgs boson, all other fermion couplings are ignored. Including the top quark (assuming $M_{t}=$ $175 \mathrm{GeV}$ ) and the gauge bosons leads to the bound $M_{H}<170 \mathrm{GeV}$ [10]. If a Higgs boson heavier than this bound were found, it would require a cutoff, $\Lambda<10^{19} \mathrm{GeV}$, and hence new physics which occurs below the Planck scale.

A lower bound on the Higgs mass can also be found from the requirement that electroweak symmetry breaking actually occurs [11]. This requirement is $V(v)<$ $V(0)$, which is essentially equivalent to the requirement that $\lambda>0$ at all scales $\Lambda$. Including the one-loop contributions to equation (1.25) due to the top quark and gauge bosons gives, for small values of $\lambda$ (and hence small $M_{H}$ ),

$$
\frac{d \lambda}{d t}=\frac{1}{16 \pi^{2}}\left[-12 g_{t}^{4}+\frac{3}{16}\left(2 g^{4}+\left(g^{2}+g^{2}\right)^{2}\right)\right]
$$


where $g_{t}=-M_{t} / v[6]$. This is easily solved to find

$$
\lambda(\Lambda)=\lambda(v)+\frac{1}{16 \pi^{2}}\left[-12 g_{t}^{4}+\frac{3}{16}\left(2 g^{4}+\left(g^{2}+g^{2}\right)^{2}\right)\right] \ln \left(\frac{\Lambda^{2}}{v^{2}}\right) .
$$

Requiring that $\lambda(\Lambda)>0$ leads to a lower bound on the Higgs mass

$$
M_{H}^{2}>\frac{v^{2}}{8 \pi^{2}}\left[-12 g_{t}^{4}+\frac{3}{16}\left(2 g^{4}+\left(g^{2}+g^{2}\right)^{2}\right)\right] \ln \left(\frac{\Lambda^{2}}{v^{2}}\right) .
$$

If the two-loop renormalization group improved effective potential is used (which sums all potentially large logarithms) and if the running of all coupling constants is included, then this lower bound becomes

$$
M_{H}(\mathrm{GeV})>130.5+2.1\left(M_{t}-174\right) \approx 130 \mathrm{GeV}
$$

for $\Lambda=10^{19} \mathrm{GeV}$ [12]. Therefore, if the Standard Model is valid up to the Planck scale, then combining this with the triviality result, the mass of the Higgs boson is constrained to the range $130 \mathrm{GeV}<M_{H}<175 \mathrm{GeV}$. However, as will be seen in section 1.4, $\Lambda$ may be much lower than the Planck scale, in which case the Standard Model must be modified by some unknown type of new physics at this scale so that a light Higgs boson can be generated.

The Higgs boson enters into one loop radiative corrections in the Standard Model and thus, precision electroweak measurements can also give bounds on the 
Higgs mass. For example, the radiative corrections to $M_{W}$ can be written as

$$
\frac{M_{W}^{2}}{M_{Z}^{2}}=1-\frac{\pi \alpha}{\sqrt{2} G_{F} M_{W}^{2}(1-\delta r)}
$$

where $\delta r$ is a function of $M_{t}^{2}$ and $\ln \left(M_{H}\right)$. Precision electroweak measurements as of September, 2005, give a 95\% confidence level upper limit on the Higgs boson mass of $M_{H}<186 \mathrm{GeV}$ [13]. If the LEP-2 direct search limit, $M_{H}>114 \mathrm{GeV}$, is included, then the upper bound increases to $M_{H}<219 \mathrm{GeV}$.

Although the Higgs boson mass remains a free parameter in the electroweak theory, the results presented in this section suggest that the Higgs boson may be relatively light, on the order of a few hundred GeV as shown in Figure 1.1 [14].

\subsection{Motivation for Physics Beyond the Standard Model}

Most theorists believe that the Higgs mechanism of the Standard Model is not entirely responsible for electroweak symmetry breaking. For example, the Higgs mechanism does not explain why $v=246 \mathrm{GeV}$ and does not explain why fermions have the masses that they do. However, perhaps the most serious problem with the Higgs mechanism is that loop corrections to the square of the Higgs mass contain a quadratic divergence, dependent on physics at some higher energy scale, $\Lambda$. For example, when the one-loop corrections to the square of the Higgs mass, $\delta M_{H}^{2}$, are 


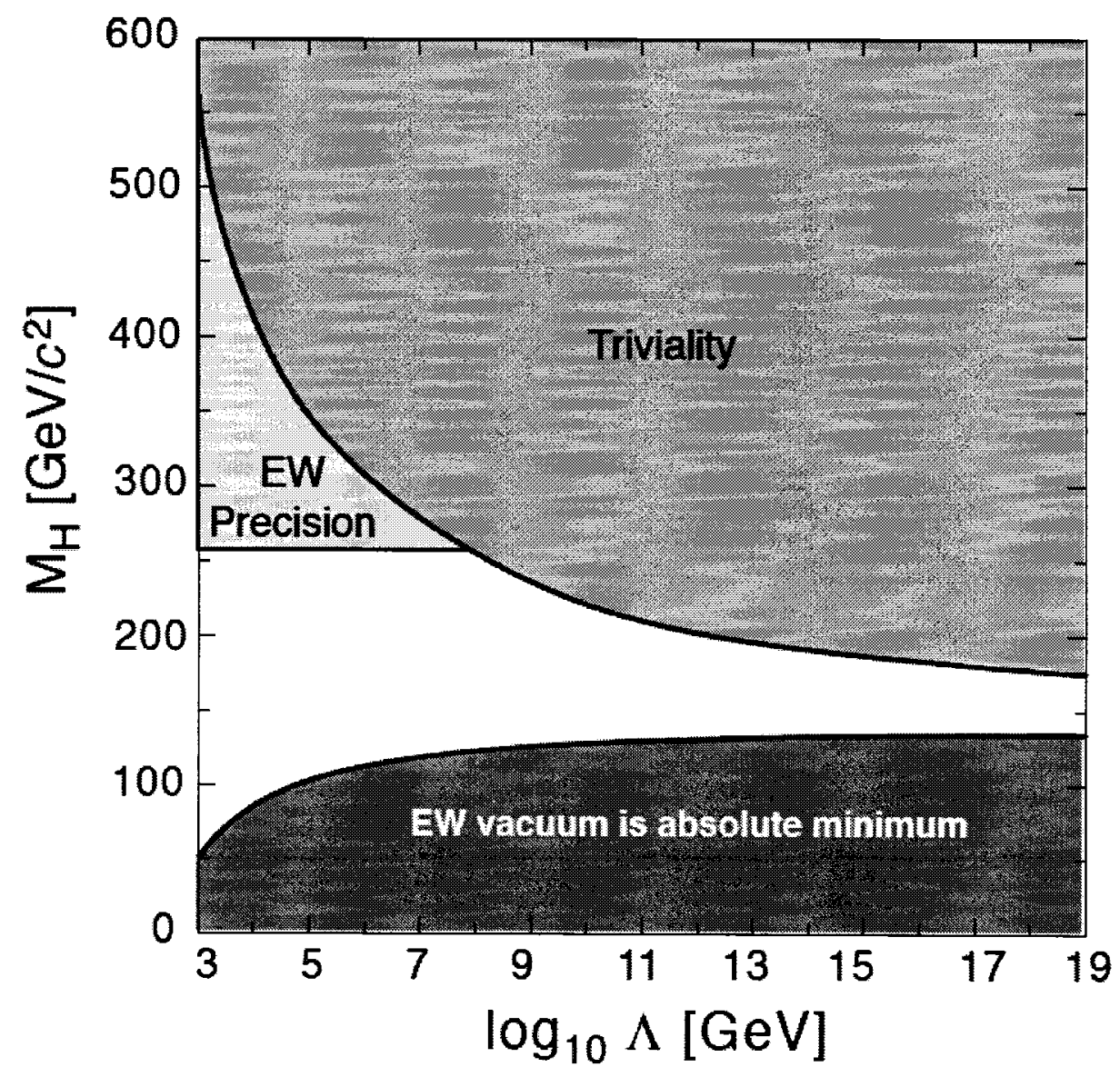

Figure 1.1: Theoretical limits on the Higgs boson mass. The upper bound follows from triviality conditions and the lower bound follows from the requirement that $V(v)<V(0)$. Also shown is the upper limit permitted by precision electroweak measurements at the $95 \%$ confidence level. The range of allowed Higgs masses shown by the white region predicts a light Higgs boson [14]. 
calculated, the result is

$$
\begin{aligned}
\delta M_{H}^{2} & =\frac{G_{F} \Lambda^{2}}{4 \sqrt{2} \pi^{2}}\left(6 M_{W}^{2}+3 M_{Z}^{2}+M_{H}^{2}-12 M_{t}^{2}\right)+\ldots \\
& \sim-\left(\frac{\Lambda}{0.7 T e V} 200 \mathrm{GeV}\right)^{2}+\ldots
\end{aligned}
$$

where the additional terms in (1.35) are not divergent in $\Lambda$ [15]. At each order in perturbation theory, counterterms could be added to cancel these divergences. The Higgs mass would then be expressed as $M_{H}^{2}=M_{H, 0}^{2}+\delta M_{H}^{2}+$ counterterms, where the counterterms must be adjusted in order to cancel the quadratically divergent contributions to $\delta M_{H}^{2}$ [6]. If the Standard Model is valid up to the Planck scale, $\Lambda=10^{19} \mathrm{GeV}$, the counterterms must be fine-tuned to many decimal places in order to cancel the divergent contributions to $\delta M_{H}^{2}$. Most theorists regard this solution as unattractive due to the absence of a natural mechanism in the Standard Model that necessitates such a large fine tuning.

One way around this problem is to fix the value of $\Lambda$ so that the Higgs mass remains light, in agreement with precision electroweak measurements. This requires that $\Lambda \sim 1 \mathrm{TeV}$, suggesting that there is some new physics at the $\mathrm{TeV}$ scale which could be responsible for generating a light Higgs boson. However, much of the possible new physics at this scale has already been excluded experimentally. A model independent analysis which examined various dimension-six operators found that new physics typically cannot occcur below a scale of roughly $\Lambda>5 \mathrm{TeV}$ [16]. This tension between needing a low scale for new physics to get a light Higgs boson, and the exclusion of possible new physics at the $\mathrm{TeV}$ scale is known as the little hierarchy problem. Several models of physics beyond the Standard Model have been proposed 
in order to solve the hierarchy problem. One such model, the Littlest Higgs model, will be described in the next section.

\subsection{The Littlest Higgs Model}

Little Higgs models [17] are a relatively new class of models for physics beyond the Standard Model that attempt to solve the little hierarchy problem. There are several variations of Little Higgs models (for recent reviews, consult [18] or [19]), such as the Simplest Little Higgs model [20], the Littlest Higgs model [21], and Little Higgs models with T-parity [22]. In this thesis, we consider the Littlest Higgs Model [21], which resolves the little hierarchy problem by introducing new particles at the $\mathrm{TeV}$ scale in addition to the particles of the Standard Model. The couplings of these particles to the Higgs boson are such that the quadratic divergences induced by the SM loops are cancelled by the quadratic divergences induced by the $\mathrm{TeV}$ scale particles at one-loop level. This cancellation occurs between particles with the same statistics: divergences due to the Standard Model gauge bosons are cancelled by the contributions of new heavy gauge bosons, and the contribution from the Higgs loop is cancelled by new heavy scalar particles. A heavy charge $\frac{2}{3}$ quark is also introduced in order to cancel the divergence from the top quark Yukawa coupling to the Higgs boson. As a result, the Standard Model Higgs boson remains light and free from one-loop quadratic sensitivity up to a cutoff scale of $\Lambda_{S} \sim 10 \mathrm{TeV}$, thus solving the little hierarchy problem. At energies greater than $\Lambda_{S}$, the model becomes strongly

coupled and new physics emerges [21]. However, the Standard Model is insensitive to the new physics of this regime. 
Electroweak Symmetry Breaking in the Littlest Higgs model is more complicated than the Higgs mechanism of the Standard Model, and proceeds as follows $[21,23]$. The Littlest Higgs model begins with an SU(5) global symmetry with a locally gauged subgroup $[\mathrm{SU}(2) \otimes \mathrm{U}(1)]^{2}$. At a scale $\Lambda_{S} \sim 4 \pi f \sim 10 \mathrm{TeV}$, the $\mathrm{SU}(5)$ global symmetry is broken down to its subgroup $\operatorname{SO}(5)$ via a vev of order $f \sim 1 \mathrm{TeV}$. At the same time, the gauge symmetry $[\mathrm{SU}(2) \otimes \mathrm{U}(1)]^{2}$ is broken down to its diagonal subgroup $S U(2)_{L} \otimes U(1)_{Y}$, identified as the electroweak gauge group of the Standard Model. The symmetry breaking leaves 14 massless Goldstone bosons, corresponding to the broken generators. Under $S U(2)_{L} \otimes U(1)_{Y}$, these transform as

$$
\mathbf{1}_{0} \oplus \mathbf{3}_{0} \oplus \mathbf{2}_{ \pm \frac{1}{2}} \oplus \mathbf{3}_{ \pm 1}
$$

The real singlet, $\mathbf{1}_{0}$, and the real triplet, $\mathbf{3}_{0}$, become the longitudinal components of the new heavy gauge bosons which acquire masses of the order $f$. The complex doublet, $\mathbf{2}_{\frac{1}{2}}$, is identified as the Standard Model Higgs boson, and $\mathbf{3}_{1}$ is an additional complex triplet of heavy Higgs bosons. At this stage of the symmetry breaking, these Higgs fields remain massless.

The Goldstone bosons can be parameterized by a non-linear sigma model field

$$
\Sigma=e^{i \Pi / f} \Sigma_{0} e^{i \Pi^{T} / f}
$$

where $\Sigma_{0}=\langle\Sigma\rangle \sim f$ is the vev associated with the symmetry breaking $S U(5) \rightarrow$ 
$S O(5)$, and the Goldstone boson matrix, $\Pi$, is given by

$$
\Pi=\left(\begin{array}{ccc} 
& h^{\dagger} / \sqrt{2} & \phi^{\dagger} \\
h / \sqrt{2} & & h^{*} / \sqrt{2} \\
\phi & h^{T} / \sqrt{2} &
\end{array}\right) .
$$

The scalar field content consists of a doublet, $h$, and a triplet, $\phi$, under the unbroken $S U(2)_{L} \otimes U(1)_{Y}$ Standard Model gauge group:

$$
h=\left(h^{+}, h^{0}\right), \quad \phi=\left(\begin{array}{cc}
\phi^{++} & \frac{\phi^{+}}{\sqrt{2}} \\
\frac{\phi^{+}}{\sqrt{2}} & \phi^{0}
\end{array}\right) .
$$

In this thesis, we are interested in the scalar sector of the Littlest Higgs model, particularly the triplet, which consists of a neutral, a singly-charged, and a doublycharged state. At this stage in the symmetry breaking, however, the scalar doublet and triplet remain massless. The masses of these particles are generated through a second step in the symmetry breaking, which will be described below.

In the Littlest Higgs model, the global symmetries prevent the appearance of a Higgs potential at tree-level. Instead, the gauge and Yukawa couplings that break the global $\mathrm{SO}(5)$ symmetry will induce a Higgs potential at one-loop and higher orders. This potential, named the Coleman-Weinberg potential [24], is given by [23]

$$
V=\lambda_{\phi^{2}} f^{2} \operatorname{Tr}\left(\phi^{\dagger} \phi\right)+i \lambda_{h \phi h} f\left(h \phi^{\dagger} h^{T}-h^{*} \phi h^{\dagger}\right)-\mu^{2} h h^{\dagger}+\lambda_{h^{4}}\left(h h^{\dagger}\right)^{2}
$$

where we have neglected terms involving $\phi^{4}$ and $h^{2} \phi^{2}$ since their contribution is small. 
For $\mu^{2}>0$, this scalar potential triggers electroweak symmetry breaking, resulting in vacuum expectation values for the doublet and triplet fields: $\left\langle h^{0}\right\rangle=v / \sqrt{2}$ and $\left\langle i \phi^{0}\right\rangle=v^{\prime}[23]$, with

$$
v^{2}=\frac{\mu^{2}}{\lambda_{h^{4}}-\lambda_{h \phi h}^{2} / \lambda_{\phi^{2}}}, \quad v^{\prime}=\frac{\lambda_{h \phi h}}{2 \lambda_{\phi^{2}}} \frac{v^{2}}{f}
$$

Minimizing the potential also leads to the relation

$$
\frac{\lambda_{h \phi h}}{\lambda_{h^{4}}}=\frac{4 \lambda_{h \phi h}}{\lambda_{\phi^{2}}}=\frac{8 v^{\prime}}{v} \frac{f}{v}
$$

The Higgs doublet and triplet states acquire masses, which to leading order, are given by $[23]$

$$
M_{\Phi}^{2} \simeq \lambda_{\phi^{2}} f^{2}, \quad M_{H}^{2} \simeq 2\left(\lambda_{h^{4}}-\lambda_{h \phi h}^{2} / \lambda_{\phi^{2}}\right) v^{2}=2 \mu^{2}
$$

Here, $H$ and $\Phi$ indicate the mass eigenstates of the scalars, as opposed to the gauge eigenstates $h$ and $\phi$. It is interesting to note that to leading order, the triplet states are degenerate in mass. Using equations (1.41) and (1.42) to solve for the couplings allows us to express the triplet mass in terms of the physical parameters $f, M_{H}^{2}, v$ and $v^{\prime}$ as

$$
M_{\Phi}^{2}=\frac{2 M_{H}^{2} f^{2}}{v^{2}} \frac{1}{\left[1-\left(4 v^{\prime} f / v^{2}\right)^{2}\right]} .
$$

As an aside, by demanding that the triplet mass squared is positive definite, we arrive at the relation between the doublet and triplet vevs:

$$
\frac{v^{\prime 2}}{v^{2}}<\frac{v^{2}}{16 f^{2}}
$$


For $f \sim 1 \mathrm{TeV}$, this gives an upper bound

$$
\frac{v^{\prime}}{v}<0.05
$$

The states of the heavy Higgs triplet can be produced at the Large Hadron Collider through vector boson fusion. In particular, the singly-charged state, $\Phi^{+}$, can be produced through $W Z$ scattering. This is an interesting channel with a charged Higgs, as the process $W^{+} Z \rightarrow \Phi^{+} \rightarrow W^{+} Z$ does not exist in two Higgs doublet models such as Supersymmetry [25]. Therefore, if this process is experimentally observed, it would provide evidence for the existence of a Higgs triplet. The Littlest Higgs model is one such Higgs triplet model, so the $W Z$ scattering signal in this model will be discussed in Chapter 2. 


\section{Chapter 2}

\section{The WZ Scattering Signal}

Experiments at the Large Hadron Collider (LHC) will study proton-proton collisions with centre of mass energies up to $\sqrt{s}=14 \mathrm{TeV}$. These experiments will produce a large number of intermediate vector bosons, $W^{ \pm}$and $Z$. As discussed in Section 1.5, we are interested in the production of the heavy Higgs bosons of the Littlest Higgs model. At the LHC, the dominant production mechanism of the singlycharged Higgs, $\Phi^{ \pm}$, is $W Z$ scattering. This process is illustrated in Figure 2.1. The incoming quarks from the colliding protons emit $W$ and $Z$ bosons, which interact with each other. The $W$ and $Z$ bosons emerge in the final state, along with two spectator quark jets. In order to simplify the calculation of the signal cross section, we can apply two approximations. These are the Effective Vector Boson Approximation (EVBA) and the Goldstone Equivalence Theorem, and are described in this chapter. 


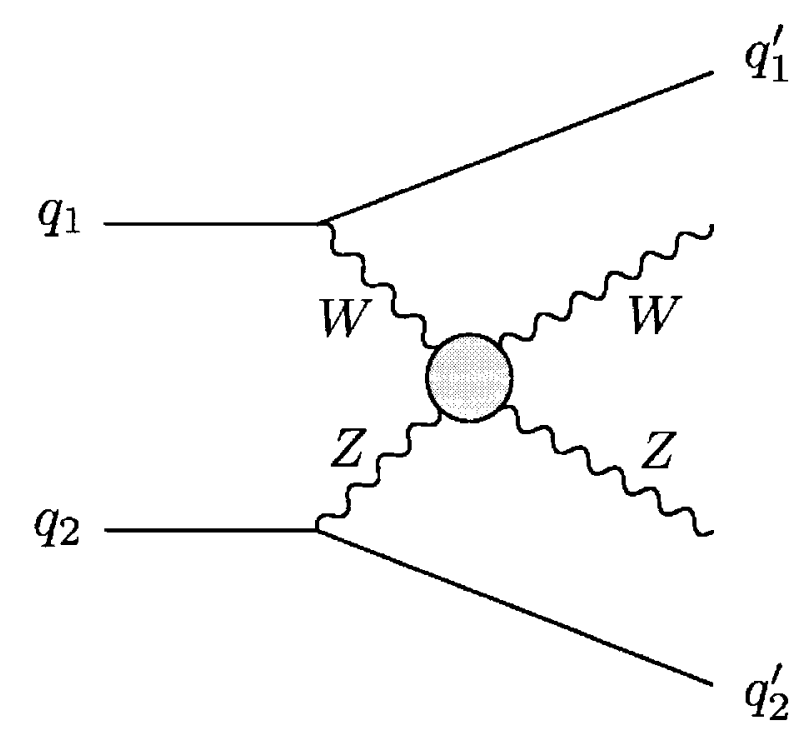

Figure 2.1: $W Z$ scattering at the LHC. The incoming quarks from the colliding protons emit $W$ and $Z$ bosons, which interact with each other. The $W$ and $Z$ bosons emerge in the final state, along with two spectator quark jets. 


\subsection{Longitudinally Polarized Vector Bosons}

The heavy Higgs bosons in the Littlest Higgs model acquire masses at the $\mathrm{TeV}$ scale due to electroweak symmetry breaking, which is triggered at a scale $\Lambda_{S} \sim 10 \mathrm{TeV}$ at which the theory becomes strongly interacting [17]. Therefore, we are interested in the production of the $\Phi^{+}$via the scattering of longitudinal gauge bosons, $W_{L} Z_{L}$, since it is these components that may interact strongly [26]. The production of transversely polarized vector bosons occurs through standard electroweak mechanisms, unrelated to the strong symmetry breaking effects. It is therefore important to understand the difference between longitudinal and transverse polarizations of the vector bosons.

The two transverse polarizations for a vector boson with four-momentum $p^{\mu}=$ $(E, 0,0,|\mathbf{p}|)$ and mass $M_{V}$, with $E^{2}=\mathbf{p}^{2}+M_{V}^{2}$, are given by

$$
\begin{aligned}
& \epsilon_{T_{1}}^{\mu}=(0,1,0,0) \\
& \epsilon_{T_{2}}^{\mu}=(0,0,1,0) .
\end{aligned}
$$

After electroweak symmetry breaking, the $W$ and $Z$ bosons acquire masses as the corresponding Goldstone bosons are eaten, giving them a longitudinal polarization in addition to the two transverse degrees of freedom:

$$
\epsilon_{L}^{\mu}=\frac{1}{M_{V}}(|\mathbf{p}|, 0,0, E)
$$

Since the photon remains massless after electroweak symmetry breaking, it does not acquire a longitudinal polarization. 
In our calculations for $W_{L} Z_{L}$ scattering, it is useful to express the longitudinal polarization vectors in terms of the four-momenta of the gauge bosons. In our notation, we define the four-momenta of the incoming $W$ and $Z$ as $p_{1}^{\mu}$ and $p_{2}^{\mu}$, respectively. Similary, we define the four-momenta of the outgoing $W$ and $Z$ as $p_{3}^{\mu}$ and $p_{4}^{\mu}$, respectively. In the $W Z$ centre of mass frame, these are given by

$$
\begin{aligned}
p_{1}^{\mu} & =\left(E_{1}, 0,0,|\mathbf{p}|\right) \\
p_{2}^{\mu} & =\left(E_{2}, 0,0,-|\mathbf{p}|\right) \\
p_{3}^{\mu} & =\left(E_{1}, 0,|\mathbf{p}| \sin \theta,|\mathbf{p}| \cos \theta\right) \\
p_{4}^{\mu} & =\left(E_{2}, 0,-|\mathbf{p}| \sin \theta,-|\mathbf{p}| \cos \theta\right)
\end{aligned}
$$

where $\theta$ is the scattering angle in the $W Z$ centre of mass frame, and

$$
\begin{aligned}
& E_{1}^{2}=\mathbf{p}^{2}+M_{W}^{2} \\
& E_{2}^{2}=\mathbf{p}^{2}+M_{Z}^{2}
\end{aligned}
$$

We define the corresponding longitudinal polarization vectors as

$$
\begin{aligned}
\epsilon_{1}^{\mu} & =\frac{1}{M_{W}}\left(|\mathbf{p}|, 0,0, E_{1}\right) \\
\epsilon_{2}^{\mu} & =\frac{1}{M_{Z}}\left(|\mathbf{p}|, 0,0,-E_{2}\right) \\
\epsilon_{3}^{\mu} & =\frac{1}{M_{W}}\left(|\mathbf{p}|, 0, E_{1} \sin \theta, E_{1} \cos \theta\right) \\
\epsilon_{4}^{\mu} & =\frac{1}{M_{Z}}\left(|\mathbf{p}|, 0,-E_{2} \sin \theta,-E_{2} \cos \theta\right) .
\end{aligned}
$$

Expressing the longitudinal polarization vectors of equation (2.5) in terms of the 
four-momenta of equation (2.3), gives

$$
\begin{aligned}
\epsilon_{1}^{\mu}=\frac{p_{1}^{\mu}}{M_{W}}+v_{1}^{\mu}, & v_{1}^{\mu}=\frac{E_{1}\left(\beta_{1}-1\right)}{M_{W} \sqrt{s}}\left[\left(\frac{\beta_{2}-1}{\beta_{2}}\right) p_{1}^{\mu}+\left(\frac{\beta_{1}+1}{\beta_{1}}\right) p_{2}^{\mu}\right] \\
\epsilon_{2}^{\mu}=\frac{p_{2}^{\mu}}{M_{Z}}+v_{2}^{\mu}, & v_{2}^{\mu}=\frac{E_{2}\left(\beta_{2}-1\right)}{M_{Z} \sqrt{s}}\left[\left(\frac{\beta_{2}+1}{\beta_{2}}\right) p_{1}^{\mu}+\left(\frac{\beta_{1}-1}{\beta_{1}}\right) p_{2}^{\mu}\right] \\
\epsilon_{3}^{\mu}=\frac{p_{3}^{\mu}}{M_{W}}+v_{3}^{\mu}, & v_{3}^{\mu}=\frac{E_{1}\left(\beta_{1}-1\right)}{M_{W} \sqrt{s}}\left[\left(\frac{\beta_{2}-1}{\beta_{2}}\right) p_{3}^{\mu}+\left(\frac{\beta_{1}+1}{\beta_{1}}\right) p_{4}^{\mu}\right] \\
\epsilon_{4}^{\mu}=\frac{p_{4}^{\mu}}{M_{Z}}+v_{4}^{\mu}, & v_{4}^{\mu}=\frac{E_{2}\left(\beta_{2}-1\right)}{M_{Z} \sqrt{s}}\left[\left(\frac{\beta_{2}+1}{\beta_{2}}\right) p_{3}^{\mu}+\left(\frac{\beta_{1}-1}{\beta_{1}}\right) p_{4}^{\mu}\right]
\end{aligned}
$$

where

$$
\begin{aligned}
& \beta_{1} \equiv \frac{|\mathbf{p}|}{E_{1}}=\left(1+\frac{M_{W}^{2}}{\mathbf{p}^{2}}\right)^{-\frac{1}{2}} \\
& \beta_{2} \equiv \frac{|\mathbf{p}|}{E_{2}}=\left(1+\frac{M_{Z}^{2}}{\mathbf{p}^{2}}\right)^{-\frac{1}{2}}
\end{aligned}
$$

and

$$
\mathbf{p}^{2}=\frac{1}{4 s}\left[\left(s-M_{W}^{2}-M_{Z}^{2}\right)^{2}-4 M_{W}^{2} M_{Z}^{2}\right]
$$

The longitudinal polarization vectors in equation (2.6) are expressed in terms of the four-momenta of the incoming and outgoing particles. This is useful because when calculating the $W_{L} Z_{L}$ scattering amplitudes, the result can be expressed in terms of dot products of the momentum four-vectors. Calculating these dot-products is relatively straight-forward:

$$
\begin{aligned}
& p_{1} \cdot p_{2}=p_{3} \cdot p_{4}=\frac{1}{2}\left(s-M_{W}^{2}-M_{Z}^{2}\right) \\
& p_{1} \cdot p_{4}=p_{2} \cdot p_{3}=\frac{1}{2}\left(M_{W}^{2}+M_{Z}^{2}-u\right)
\end{aligned}
$$




$$
\begin{aligned}
& p_{1} \cdot p_{3}=M_{W}^{2}-\frac{t}{2} \\
& p_{2} \cdot p_{4}=M_{Z}^{2}-\frac{t}{2}
\end{aligned}
$$

where the Mandelstam variables, $s, t$ and $u$, are given by

$$
\begin{aligned}
& s=\left(p_{1}+p_{2}\right)^{2}=\left(p_{3}+p_{4}\right)^{2} \\
& t=\left(p_{1}-p_{3}\right)^{2}=\left(p_{4}-p_{2}\right)^{2}=-2 \mathbf{p}^{2}(1-\cos \theta) \\
& u=\left(p_{1}-p_{4}\right)^{2}=\left(p_{3}-p_{2}\right)^{2}=-2 \mathbf{p}^{2}(1+\cos \theta)
\end{aligned}
$$

For the remainder of this thesis, we will denote the $W Z$ centre of mass energy by $M_{W Z}$ rather than $\sqrt{s}$. This is to avoid confusion with the proton-proton centre of mass energy which is typically denoted by $\sqrt{s}$. $M_{W Z}$ is referred to as the invariant mass of the $W Z$ pair. It is also worth mentioning that the results in this section can be applied to general two-body scattering processes of the form $V_{1} V_{2} \rightarrow V_{1} V_{2}$. These results can also be simplified if we consider the scattering of particles with equal masses. This allows us to consider not only $W_{L} Z_{L}$ scattering, but $W_{L} W_{L}$ or $Z_{L} Z_{L}$ scattering as well. However, in this thesis, we will focus on $W_{L} Z_{L}$ scattering.

\subsection{The Effective Vector Boson Approximation}

The proton is known to be composed of consituents called partons (quarks and gluons) which interact with each other independently. As higher energies are reached experimentally, it becomes important to include more partons in theoretical 
calculations. As the charm, bottom and top thresholds are reached, these heavy quarks contribute to the parton sea. At high energies in the $\mathrm{TeV}$ range, the parton sea includes the $W$ and $Z$ gauge bosons as well. By considering these bosons as partons, calculations involving gauge bosons in the intermediate states can be considerably simplified. This is the essence of the EVBA, and this will be applied to $W Z$ scattering in this section.

Using the EVBA [27], the cross section for $W Z$ scattering is given by

$$
\frac{d \sigma_{p p \rightarrow W Z}(s)}{d M_{W Z}}=\left.\frac{2 M_{W Z}}{s} \frac{d \mathcal{L}}{d \tau}\right|_{p p / W Z} \hat{\sigma}_{W Z \rightarrow W Z}(\tau s)
$$

where $\tau=M_{W Z}^{2} / s$ is the ratio of the $W Z$ invariant mass, $M_{W Z}$, to the proton-proton centre of mass energy, $\sqrt{s}=14 \mathrm{TeV}$. The parton-level cross section, $\hat{\sigma}_{W Z \rightarrow W Z}(\tau s)$, is computed for the subprocess $W Z \rightarrow W Z$, and $\left.\frac{d \mathcal{L}}{d \tau}\right|_{p p / W Z}$ is the luminosity of vector bosons in the proton-proton system, given by

$$
\left.\frac{d \mathcal{L}}{d \tau}\right|_{p p / W Z}=\left.\sum_{i j} \int_{\tau}^{1} \frac{d \tau^{\prime}}{\tau^{\prime}} \int_{\tau^{\prime}}^{1} \frac{d x}{x} f_{i}(x) f_{j}\left(\frac{\tau^{\prime}}{x}\right) \frac{d \mathcal{L}}{d \zeta}\right|_{q_{i} q_{j} / W Z}
$$

Here, $\tau^{\prime}=M_{W Z}^{2} / \hat{s}$ and $\zeta=\tau / \tau^{\prime}=\hat{s} / s$, where $\sqrt{\hat{s}}$ is the centre of mass energy of the quark-quark system. The parton distribution functions (PDFs), $f_{i}(x)$, give the probability for a quark of flavour $i$ to be found in the proton with a fraction, $x$, of the proton's total momentum. These are normalized such that

$$
\int_{0}^{1} x f_{i}(x) d x=1
$$

In this thesis, we employ the CTEQ6M set of parton distribution functions [28], which 
are implemented numerically. The CTEQ6M PDFs are shown in Figure 2.2. Note that the PDFs are dependent not only on the momentum fraction, $x$, but also on the energy scale, $\mathrm{Q}$, of the parton-level interaction. In our calculations, we use $\mathrm{Q}=M_{W Z}$.
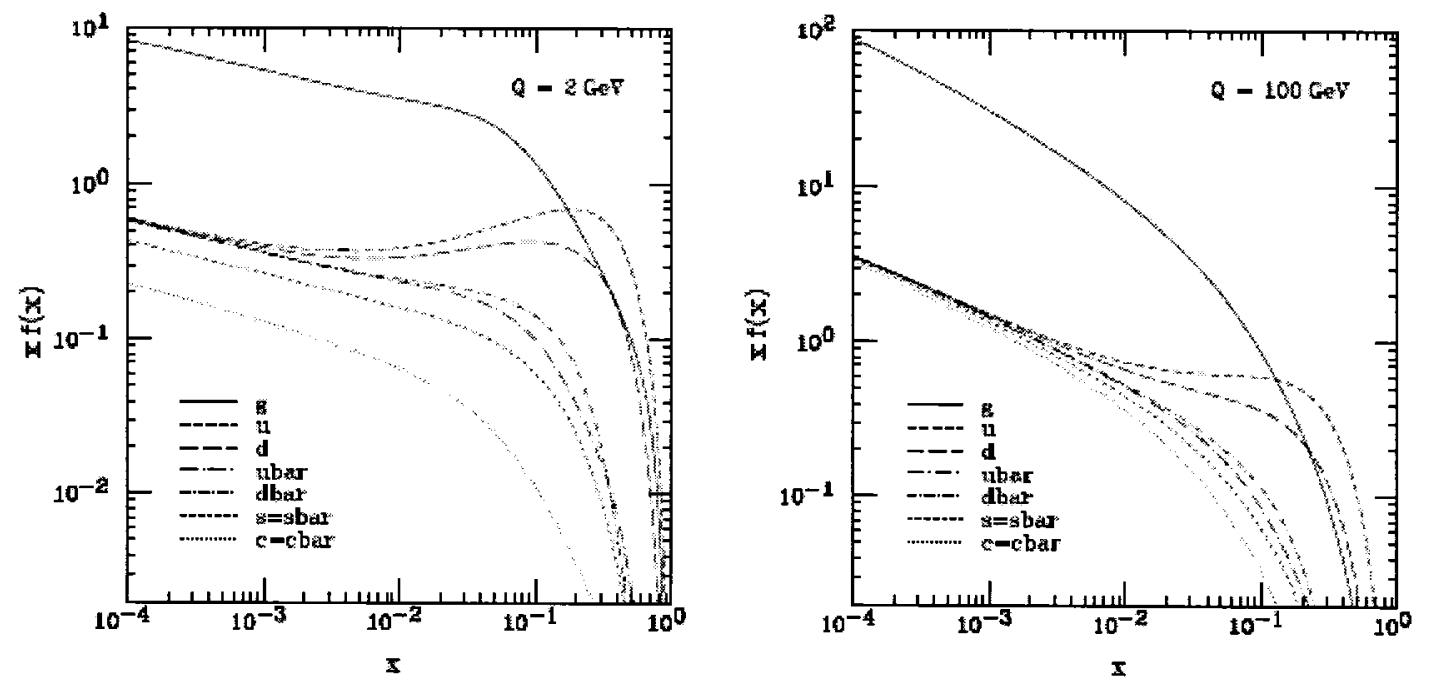

Figure 2.2: The CTEQ6M parton distribution functions at $\mathrm{Q}=2$ and $100 \mathrm{GeV}$.

The luminosity of vector bosons in the quark-quark system is given by

$$
\left.\frac{d \mathcal{L}}{d \tau}\right|_{q_{i} q_{j} / W Z}=\int_{\tau}^{1} \frac{d x}{x} f_{q_{i} / W}(x) f_{q_{j} / Z}\left(\frac{\tau}{x}\right),
$$

where $f_{q_{i} / V}(x)$ is the parton distribution function for vector boson $V$ to be found inside quark $i$. The PDFs for vector bosons in a quark are given by analytic expressions, the form of which depends on the polarization of the vector boson. For transversely 
polarized vector bosons, we have [27]

$$
f_{q / V_{T}}(x)=\frac{C_{V}^{2}+C_{A}^{2}}{8 \pi^{2} x}\left[x^{2}+2(1-x)\right] \ln \left(\hat{s} / M_{V}^{2}\right)
$$

whereas for longitudinal vector bosons, we have [27]

$$
f_{q / V_{L}}(x)=\frac{C_{V}^{2}+C_{A}^{2}}{4 \pi^{2}}\left(\frac{1-x}{x}\right)
$$

The vector and axial-vector couplings for the quarks are given by

$$
\begin{aligned}
& C_{V}=\left\{\begin{array}{cc}
\frac{g}{2 \sqrt{2}} & \text { for } V=W \\
\frac{g}{\cos \theta_{W}}\left(\frac{1}{2} T_{3}-Q_{q} \sin ^{2} \theta_{W}\right) & \text { for } V=Z
\end{array}\right. \\
& C_{A}=\left\{\begin{array}{cc}
-\frac{g}{2 \sqrt{2}} & \text { for } V=W \\
-\frac{g}{\cos \theta_{W}}\left(\frac{1}{2} T_{3}\right) & \text { for } V=Z
\end{array}\right.
\end{aligned}
$$

where $Q_{q}$ is the quark charge and $T_{3}$ is the third component of weak isospin for the quark:

$$
T_{3}=\left\{\begin{aligned}
+\frac{1}{2} & \text { for } q=u, c, t, \bar{d}, \bar{s}, \bar{b} \\
-\frac{1}{2} & \text { for } q=d, s, b, \bar{u}, \bar{c}, \bar{t}
\end{aligned}\right.
$$

As we can see from equation (1.23), the weak coupling constant, $g$, is dependent on the $W$ boson mass, $M_{W}$, and the Fermi coupling constant, $G_{F}$. Using the values $M_{W}=80.403 \pm 0.029 \mathrm{GeV}$ and $G_{F}=(1.16637 \pm 0.00001) \times 10^{-5} \mathrm{GeV}^{-2}[7]$, we obtain $g=0.653$. 
In order to apply the EVBA, we require that

$$
x \gg 2 \frac{M_{V}}{\sqrt{\hat{s}}},
$$

where $x$ is the momentum fraction carried by either quark in the proton-proton collision. In the numerical calculations, we have cut off all integrals at this minimum value of $x$. Furthermore, for resonant production of a particle with mass $M$ in vector boson scattering, the EVBA is only valid for $M \gg 2 M_{V}$. This condition is satisfied in the calculations, since we are considering production of the singly-charged heavy Higgs, $\Phi^{+}$, which has a mass $M \sim 1 T e V$.

The total cross section for $W Z$ scattering at the LHC, given by equation (2.11), can therefore be calculated by first computing the parton level cross section, $\hat{\sigma}_{W Z \rightarrow W Z}$. This is then integrated over the appropriate parton distribution functions and summed over the relevant quark flavours. The parton level cross section for $W_{L} Z_{L}$ scattering will be calculated using two methods. In Section 2.3 the parton level cross section will be calculated using longitudinally polarized vector bosons, employing the results of Section 2.1. The calculation of the parton level cross section for $W_{L} Z_{L}$ scattering can also be simplified using the Goldstone Equivalence Theorem, which will be described in Section 2.4. 


\subsection{Parton Level Cross Section for $W_{L} Z_{L}$ Scatter- ing}

The parton level cross section for $W_{L} Z_{L}$ scattering is calculated by adding the individual scattering amplitudes arising from the diagrams of Figure 2.3:

$$
\mathcal{A}\left(W_{L}^{+} Z_{L} \rightarrow W_{L}^{+} Z_{L}\right)=\mathcal{A}_{a}+\mathcal{A}_{b}+\mathcal{A}_{c}+\mathcal{A}_{d}+\mathcal{A}_{e}+\mathcal{A}_{f}+\mathcal{A}_{g}
$$

The Feynman rules required for this calculation [23] are presented in Table A.1 of Appendix A. For example, the amplitude for the s-channel $\Phi^{+}$resonance of diagram (g) from Figure 2.3 is given by

$$
\mathcal{A}_{g}=\epsilon_{1}^{\mu} \epsilon_{2}^{\nu} \epsilon_{3}^{\rho *} \epsilon_{4}^{\sigma *}\left[-i \frac{g^{2}}{c_{W}} v^{\prime} g_{\mu \nu}\right]\left[\frac{i}{M_{W Z}^{2}-M_{\Phi}^{2}+i M_{\Phi} \Gamma_{\Phi^{+}}}\right]\left[-i \frac{g^{2}}{c_{W}} v^{\prime} g_{\rho \sigma}\right]
$$

where the $\epsilon_{i}$ are the longitudinal polarization vectors given by equation (2.6). For the decay width, $\Gamma_{\Phi^{+}}$, we only included the decay $\Phi^{+} \rightarrow W^{+} Z$. Note that the amplitude for this resonant signal is proportional to the square of the triplet vev, $v^{\prime 2}$; this will be discussed in more detail later in this thesis.

Similar expressions can be written for the amplitudes of the other diagrams of Figure 2.3. The amplitudes for each diagram were calculated using Mathematica, which is capable of contracting over the indices in expressions such as equation (2.22). The longitudinal polarization vectors of equation (2.6) were used in these calculations, as well as the various kinematic expressions from Section 2.1. We find that the resulting expressions for the parton level scattering amplitudes are dependent only 


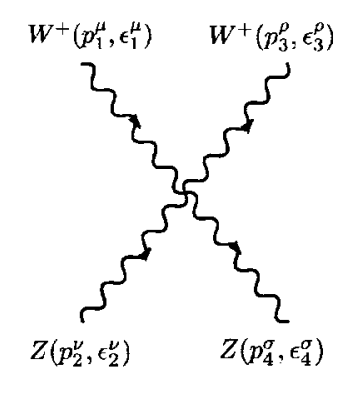

(a)

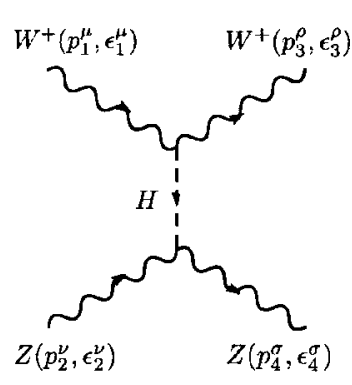

(b)

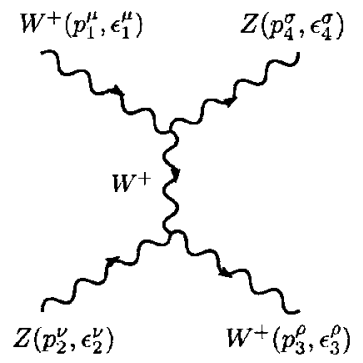

(c)

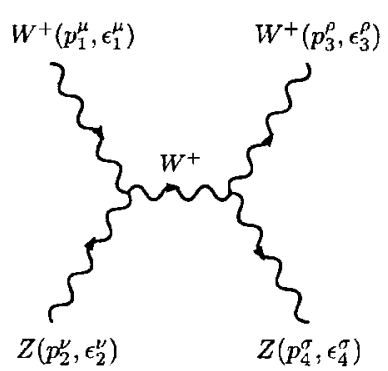

(d)

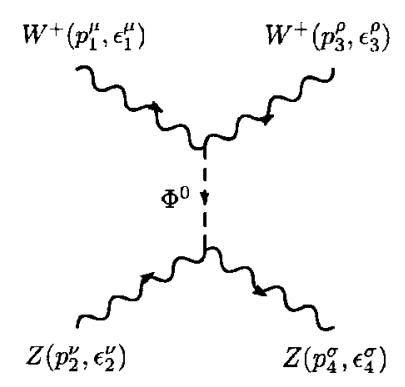

(e)

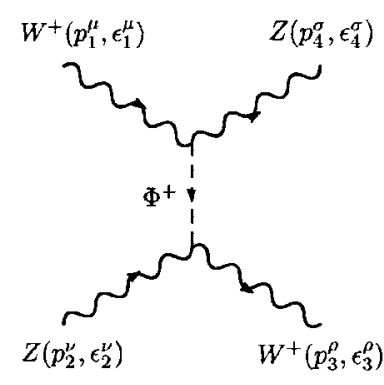

(f)

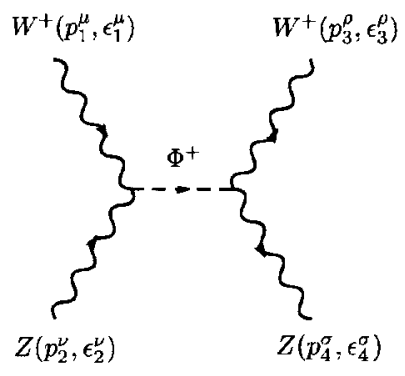

(g)

Figure 2.3: Feynman diagrams for $W Z$ scattering. In the Standard Model, only diagrams (a) - (d) are present. In the Littlest Higgs model, diagrams (e) - (g) must also be taken into account. 
on the invariant mass, $M_{W Z}$, and the $W Z$ scattering angle $z \equiv \cos \theta$. We then integrate numerically over $z$ to obtain the parton level cross section

$$
\hat{\sigma}_{W Z \rightarrow W Z}=\int_{-1}^{1} \frac{d \hat{\sigma}_{W Z \rightarrow W Z}}{d z} d z=\int_{-1}^{1} \frac{|\mathcal{A}|^{2}}{32 \pi^{2} M_{W Z}^{2}} d z
$$

We then apply the Effective Vector Boson Approximation by integrating numerically over the appropriate parton distribution functions. This allows us to plot the total cross section as a function of the invariant mass, $M_{W Z}$. This plot of $d \sigma / d M_{W Z}$ as a function of $M_{W Z}$ is called the invariant mass distribution of the $W Z$ pair.

Reference [23], performs this calculation for the process $W_{L}^{+} W_{L}^{+} \rightarrow W_{L}^{+} W_{L}^{+}$. In order to verify that the EVBA was used correctly, we first reproduced the invariant mass distribution for this process, which involves the production of the doubly-charged heavy Higgs, $\Phi^{++}$, of the Littlest Higgs model. See Figure 6 of [23]. Once we confirmed their result, we proceeded to calculate the invariant mass distribution for $W_{L} Z_{L}$ scattering.

\subsection{The Goldstone Equivalence Theorem}

The Goldstone Equivalence Theorem states that at high energy, when calculating scattering amplitudes of longitudinal gauge bosons, the external gauge bosons can be replaced by the corresponding Goldstone bosons [29]. This simplifies the calculation of the $W_{L} Z_{L}$ scattering cross section since the vector bosons are spin-one particles with three degrees of freedom, whereas the Goldstone bosons are spin-zero particles 
with only one degree of freedom. A general statement of the theorem is given by [29]

$$
\begin{aligned}
\mathcal{A}\left(V_{L}^{1} V_{L}^{2} \cdots V_{L}^{N} \rightarrow V_{L}^{1} V_{L}^{2} \cdots V_{L}^{N^{\prime}}\right)= & (i)^{N}(-i)^{N^{\prime}} \mathcal{A}\left(G_{1} G_{2} \cdots G_{N} \rightarrow G_{1} G_{2} \cdots G_{N^{\prime}}\right) \\
& +\mathcal{O}\left(\frac{M_{V}^{2}}{s}\right) .
\end{aligned}
$$

For the case of $W_{L} Z_{L}$ scattering, this simplifies to

$$
\mathcal{A}\left(W_{L}^{ \pm} Z_{L} \rightarrow W_{L}^{ \pm} Z_{L}\right)=\mathcal{A}\left(G^{ \pm} G^{0} \rightarrow G^{ \pm} G^{0}\right)+\mathcal{O}\left(\frac{M_{V}^{2}}{M_{W Z}^{2}}\right)
$$

Note that the Goldstone Equivalence Theorem requires that $M_{W Z} \gg M_{V}$. When the amplitude was computed using longitudinal $W$ and $Z$ bosons, the diagrams of Figure 2.3 were taken into account. However, when using the Goldstone Equivalence Theorem, the external longitudinal gauge bosons are replaced by the corresponding Goldstone bosons. This is shown in Figure 2.4. One should apply the Goldstone Equivalence Theorem with caution, however, as it does not work diagram by diagram. The complete set of gauge-invariant Feynman diagrams in the scattering process of interest must be taken into account. The calculation of scattering amplitudes is now considerably easier and can be carried out without the help of computer programs such as Mathematica. For example, using the Feynman rules in Table A.2, the amplitude for diagram (g) of Figure 2.4 is given by

$$
\begin{aligned}
\mathcal{A}_{g} & =\left[2 \frac{M_{\Phi}^{2} v^{\prime}}{v^{2}}\left(1-4 \frac{v^{\prime 2}}{v^{2}}\right)\right]\left[\frac{i}{M_{W Z}^{2}-M_{\Phi}^{2}+i M_{\Phi} \Gamma_{\Phi^{+}}}\right]\left[2 \frac{M_{\Phi}^{2} v^{\prime}}{v^{2}}\left(1-4 \frac{v^{2}}{v^{2}}\right)\right] \\
& =\frac{4 M_{\Phi}^{4} v^{\prime 2}}{v^{4}}\left(1-\frac{4 v^{\prime 2}}{v^{2}}\right)^{2}\left[\frac{i}{M_{W Z}^{2}-M_{\Phi}^{2}+i M_{\Phi} \Gamma_{\Phi^{+}}}\right] .
\end{aligned}
$$




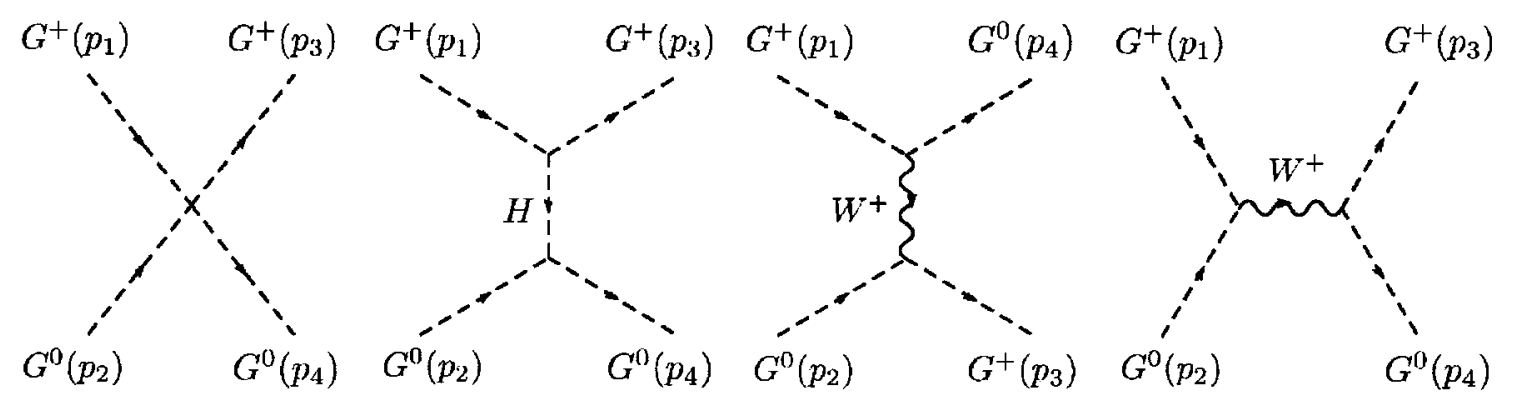

(a)

(b)

(c)

(d)

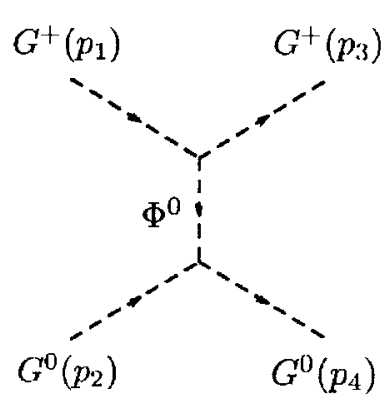

(e)

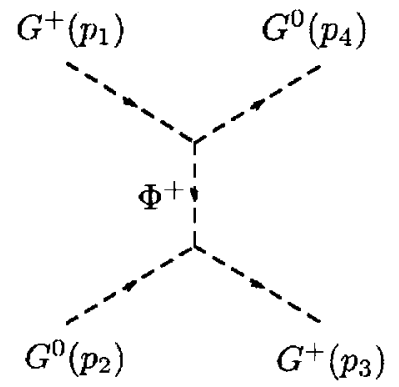

(f)

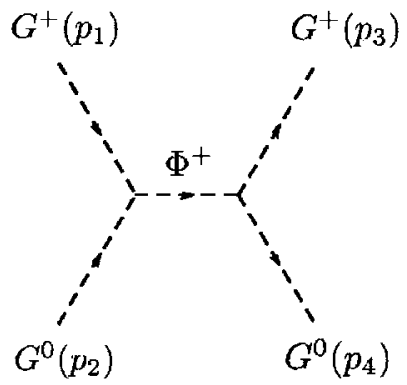

(g)

Figure 2.4: Feynman diagrams for $W_{L} Z_{L}$ scattering in the Littlest Higgs model using the Goldstone Equivalence Theorem. The external longitudinal gauge bosons of Figure 2.3 have been replaced by the corresponding unphysical Goldstone bosons. 
Similar expressions for the other diagrams are easily obtained. The resulting parton level cross section was calculated and, using the EVBA once again, the $W_{L} Z_{L}$ invariant mass distribution was plotted.

Figure 2.5 shows the invariant mass distribution for the $W_{L} Z_{L}$ scattering signal at the LHC, calculated using the EVBA. The parton-level scattering amplitudes were calculated using both external longitudinal gauge bosons, and the Goldstone Equivalence Theorem. A Higgs triplet mass of $M_{\Phi}=1.5 \mathrm{TeV}$ was used in the calculations, along with a decay width of $\Gamma_{\Phi}=10 \mathrm{GeV}$. The values of the other parameters were chosen to be $M_{H}=120 \mathrm{GeV}, v^{\prime} / v=0.05$ and $f=1070 \mathrm{GeV}$ (this value of $f$ was constrained by Equation (1.44) after choosing the other parameters).

The dotted curve in Figure 2.5 shows the result for $W_{L} Z_{L}$ scattering in the Standard Model, whereas the other two curves are for $W_{L} Z_{L}$ scattering in the Littlest Higgs model. The solid curve was obtained using external longitudinal gauge bosons and the dashed curve was obtained using the Goldstone Equivalence Theorem. Note that the two curves agree quite well, especially at large values of $M_{W Z}$. This is expected since, at high invariant mass, the calculation using the Goldstone Equivalence Theorem should agree more closely with the calculation using longitudinally polarized vector bosons. Now that the signal has been calculated, we must consider backgrounds to determine whether this process can be experimentally observed. This is the focus of Chapter 3 . 


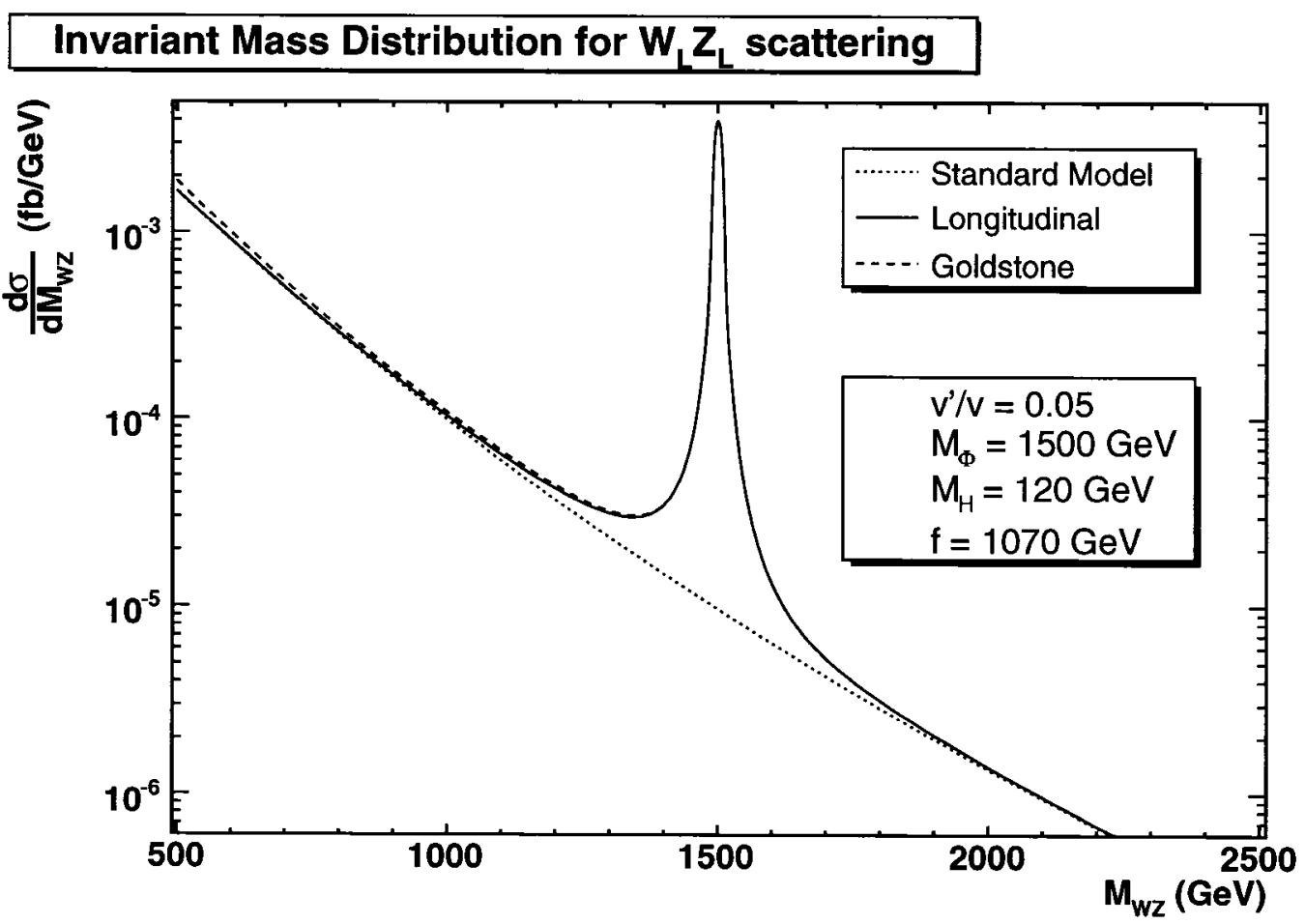

Figure 2.5: Invariant mass distribution for $W_{L} Z_{L}$ scattering. The dotted curve shows the result for $W_{L} Z_{L}$ scattering in the Standard Model and the other two curves are for $W_{L} Z_{L}$ scattering in the Littlest Higgs model. The solid curve was obtained using longitudinally polarized gauge bosons and the dashed curve was obtained using the Goldstone Equivalence Theorem. These two curves agree quite well, especially at large values of $M_{W Z}$, as expected. 


\section{Chapter 3}

\section{Backgrounds to WZ Scattering}

In Chapter 2 we demonstrated that at high energy, the cross section for $W Z$ scattering is dominated by longitudinally polarized gauge bosons. We therefore calculated the cross section for the $W_{L} Z_{L}$ scattering signal in the Littlest Higgs model at the LHC. To determine whether this process can be experimentally observed, we must now consider backgrounds to this process. Backgrounds are processes which have the same final state particles as the signal process. However, the kinematic distributions of the final state particles tend to be different for the signal and backgrounds. In order to improve the signal to background ratio, we must therefore examine these distributions and impose kinematic cuts on the final state particles that will reduce the backgrounds but not the signal. This analysis was carried out with the help of the WHIZARD event generator [30]. Unfortunately, using WHIZARD, it is not possible to separate the contributions from the longitudinal and transverse polarizations of the $W$ and $Z$ bosons. We must therefore consider $W Z$ scattering rather than $W_{L} Z_{L}$ 
scattering for our WHIZARD analysis.

\subsection{Description of Backgrounds}

We will consider three types of backgrounds to the $p p \rightarrow W Z X$ signal following the treatment of references [31-33]. The first of these backgrounds, which we denote as the electroweak background, is shown in Figure 3.1. This is a background to $W_{L} Z_{L}$ scattering, and involves $W Z$ production through vector boson fusion in which at least one of the vector bosons is transversely polarized: $p p \rightarrow W_{T} Z_{L} X, p p \rightarrow W_{L} Z_{T} X$ and $p p \rightarrow W_{T} Z_{T} X$. The electroweak background also includes diagrams in which $W$ and $Z$ bosons are radiated by the incoming quarks via electroweak interactions. Note that spectator quark jets are present in the final state. This process is a background in the sense that its cross section is essentially insensitive to the new $\mathrm{TeV}$ scale physics of the Littlest Higgs model. The electroweak background is the most difficult background to reduce; it is irreducible in the sense that a $W$ and $Z$ are produced through the same diagrams as the signal process, differing only by their polarization.

The second type of background process that we will consider is the di-boson production background of Figure 3.2, which arises from $q \bar{q}$ annihilation. Due to the absence of spectator quark jets, this background has a very different final state structure than the signal. Therefore, by requiring a forward tag jet, the di-boson production background can be greatly suppressed. This will be discussed in greater detail in Section 3.3.

Lastly, we consider backgrounds from heavy quark processes, especially those 

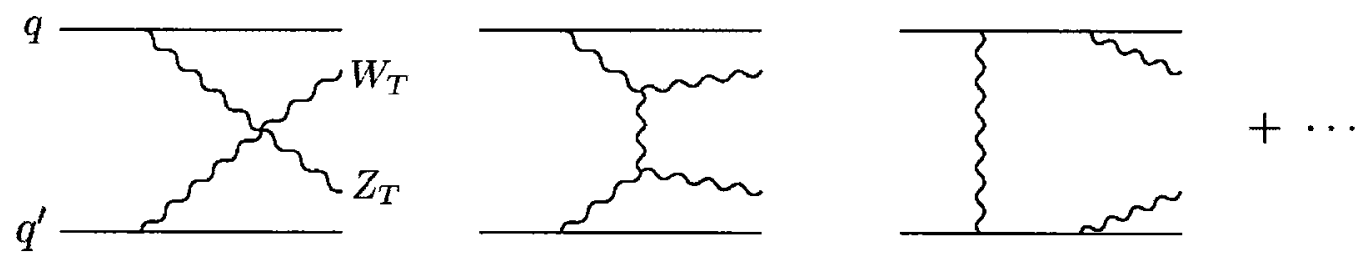

Figure 3.1: The electroweak background to $W_{L} Z_{L}$ scattering, in which at least one of the vector bosons is transversely polarized. The electroweak background also includes diagrams in which $W$ and $Z$ bosons are radiated by the incoming quarks via electroweak interactions. Note that this irreducible background contains spectator quark jets in the final state.
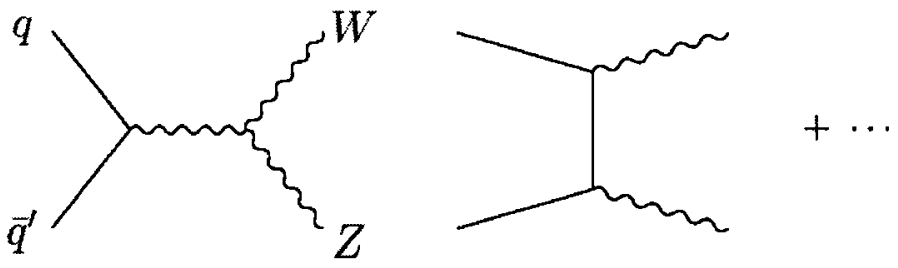

Figure 3.2: The di-boson production background. This background does not involve any quark jets and can be suppressed by requiring a forward jet tag.
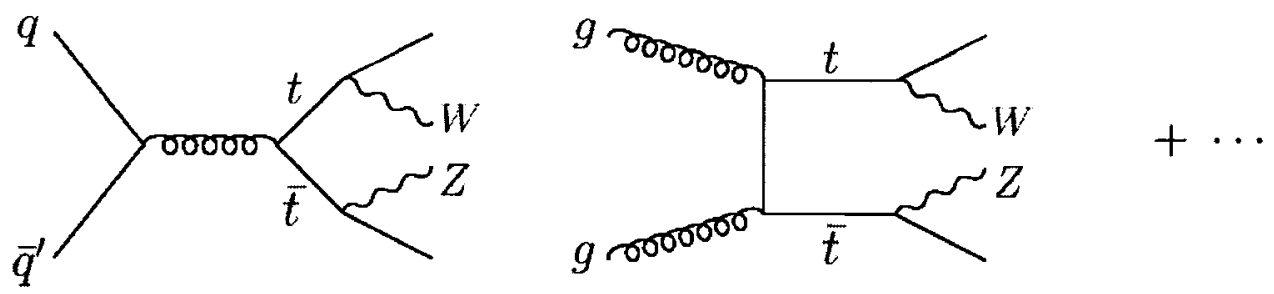

Figure 3.3: Backgrounds from heavy quark processes, arising from $t \bar{t}$ production followed by $t$ and $\bar{t}$ decays to real $W$ and $Z$ bosons. The jets in this background process tend to be more central than those of the signal, so this background can be suppressed by imposing a central jet veto. 
involving top quarks. This background arises from $t \bar{t}$ production followed by $t$ and $\bar{t}$ decays to real $W$ and $Z$ bosons as shown in Figure 3.3. These top quark background processes also involve jets in the final state. Fortunately, unlike the jets from the signal, they tend to emerge in the central regions of the detector. This background can therefore be suppressed by imposing a central jet veto, which will be further discussed in Section 3.3.

One also finds that a forward jet tag and central jet veto are effective in reducing the electroweak background. However, since this background is irreducible, it cannot be completely removed without substantially reducing the signal; even after appropriate kinematic cuts, an electroweak background remnant is still present.

The $W$ and $Z$ bosons quickly decay after being produced at the LHC, and the decay products are detected in the detector. Hadronic decays, in which the $W$ and $Z$ bosons decay to quarks, have the highest branching ratio. However, in this thesis we consider the purely leptonic decay modes, namely, the "gold-plated" events, with $W^{+} \rightarrow \ell^{+} \nu_{\ell}$ and $Z \rightarrow \ell^{+} \ell^{-}(\ell=e, \mu)$. This provides a cleaner signal than hadronic

decays, but at the cost of a much smaller branching ratio $B R\left(W^{+} Z \rightarrow \ell^{+} \nu_{\ell} \ell^{+} \ell^{-}\right) \approx$ 1.5\%. However, since the neutrino leaves the detector undetected, the final state cannot be fully reconstructed.

\subsection{The WHIZARD Event Generator}

WHIZARD is a Monte Carlo integration and event generation package to calculate cross sections for arbitrary partonic processes [30]. This is accomplished by 
calling external programs such as CompHEP [34], MadGraph [35] and O'Mega [36], which generate tree-level matrix elements for the process of interest. WHIZARD then performs an adaptive multi-channel method for the phase space integration, allowing the program to calculate numerically stable cross sections. Event samples can then be generated and analyzed.

In our calculations involving WHIZARD, we used the O'Mega matrix element generator rather than CompHEP or MadGraph. This is because O'Mega can generate matrix elements for an arbitrary number of final state particles, whereas CompHEP only supports processes with up to four final state particles. For example, the $W Z$ scattering process $q_{1} q_{2} \rightarrow q_{1}^{\prime} q_{2}^{\prime} \ell^{+} \nu_{\ell} \ell^{+} \ell^{-}$clearly involves six final state particles. MadGraph also supports six-particle final states, but does not allow flavour summation. Parton distribution functions can be accounted for by calling the PDFLIB module which is included with the WHIZARD source code. Therefore, processes arising from proton-proton collisions at the LHC can be easily simulated. A variety of models can be implemented in WHIZARD; in particular, background processes are simulated using the Standard Model and our signal process for $W Z$ scattering is simulated in the Littlest Higgs model.

It should be noted that WHIZARD does not use the Effective Vector Boson Approximation or the Goldstone Equivalence Theorem when calculating the cross section. WHIZARD instead uses the full set of tree level Feynman diagrams for the process of interest. Before starting the integration, a phase space configuration is generated automatically by WHIZARD. The phase space configuration is then adapted until a sufficiently stable estimate for the cross section is achieved. This is 
followed by the integration step, during which the cross section is calculated. Events can then be generated and kinematic distributions of the final state particles can be plotted.

In our analysis of WZ scattering using WHIZARD, we used the following input parameters: $G_{F}=1.16637 \times 10^{-5} \mathrm{GeV}^{-2}, M_{W}=80.403 \mathrm{GeV}, M_{Z}=91.1876 \mathrm{GeV}$ and $M_{H}=120 \mathrm{GeV}$. The additional parameters of the Standard Model are easily derived from these values; using equation (1.23) we get $v=246 \mathrm{GeV}$ and $g=0.653$.

We also obtain $\sin ^{2} \theta_{W}=1-\left(\frac{M_{W}}{M_{Z}}\right)^{2}=0.223$. The additional parameters in the Littlest Higgs Model were chosen to be $f=1 \mathrm{TeV}, v^{\prime} / v=0.2$ and $\Gamma_{\Phi^{+}}=10 \mathrm{GeV}$. Heavy Higgs triplet masses of $M_{\Phi}=1 \mathrm{TeV}, M_{\Phi}=1.5 \mathrm{TeV}$ and $M_{\Phi}=2 \mathrm{TeV}$ were investigated. The centre of mass energy for the LHC was chosen to be $\sqrt{s}=14 \mathrm{TeV}$, with an integrated luminosity of $\mathcal{L}=100 \mathrm{fb}^{-1}$.

\subsection{Kinematics of Final State Particles}

We now discuss the experimental signatures of the signal and background processes and examine the kinematic distributions of the final state particles. This will help us impose cuts on these kinematic variables in order to improve the signal to background ratio. Before we proceed, we must first define a few of these kinematic variables.

The transverse momentum, $p_{T}$, and longitudinal momentum, $p_{L}$, of a particle 
are given by

$$
\begin{aligned}
& p_{T}=|\mathbf{p}| \sin \theta \\
& p_{L}=|\mathbf{p}| \cos \theta \\
& \mathbf{p}^{2}=p_{T}^{2}+p_{L}^{2}
\end{aligned}
$$

where $\mathbf{p}$ is the particle's three-momentum and $\theta$ is the angle of the particle's motion with respect to the beam axis. In our $W Z$ signal and background processes, the neutrino arising from the decay of the $W$ boson passes through the detector undetected. This prevents us from fully reconstructing the final state, since the total momentum of the neutrino cannot be determined. However, since we assume that the initial transverse momentum of the entire system is zero, the missing transverse momentum, $p_{T}^{\text {miss }}$ (which is identified with the neutrino's $p_{T}$ ), can be inferred from the measured $p_{T}$ of the charged leptons and jets. We can therefore use transverse momentum as a kinematic variable when applying cuts on the final state particles. Since the $W$ boson cannot be fully reconstructed, we cannot determine the invariant mass of the $W Z$ pair. However, we can examine the $W Z$ cluster transverse mass defined by

$$
M_{T}^{2}=\left[\sqrt{M^{2}(\ell \ell \ell)+p_{T}^{2}(\ell \ell \ell)}+\left|\mathbf{p}_{T}^{m i s s}\right|\right]^{2}-\left[\mathbf{p}_{T}(\ell \ell \ell)+\mathbf{p}_{T}^{m i s s}\right]^{2}
$$

The background processes to $W Z$ scattering were generated using WHIZARD without imposing any kinematic cuts, and the transverse mass distributions for each type of background are plotted in Figure 3.4. An integrated luminosity of $\mathcal{L}=$ 
$100 \mathrm{fb}^{-1}$ was used, and relates the cross section, $\sigma$, to the number of events generated, $N$, by

$$
N=\mathcal{L} \sigma
$$

We can also define the rapidity, $y$, as

$$
y=\frac{1}{2} \ln \left(\frac{E+p_{L}}{E-p_{L}}\right)
$$

It can be shown that for $|\mathbf{p}| \gg m$, the rapidity can be expressed as

$$
y \approx-\ln \left[\tan \left(\frac{\theta}{2}\right)\right]
$$

Therefore, using this equation, we see that small values of $y$ correspond to central regions of the detector $(\theta$ near $\pi / 2)$. Similarly, we see that large values of $y$ correspond to the forward/backward regions of the detector $(\theta$ near 0 or $\pi$ ). Therefore, when applying a forward jet tag or a central jet veto, rapidity is a useful variable to consider.

For our analysis of $W Z$ scattering using WHIZARD, we define the signal process as $p p \rightarrow W^{+} Z X$ and we consider the gold-plated decay modes $W^{+} \rightarrow \ell^{+} \nu_{\ell}$ and $Z \rightarrow \ell^{+} \ell^{-}(\ell=e, \mu)$. The experimental signature is given by three isolated, charged leptons in the central rapidity region of the detector, with large transverse momentum. This is accompanied by a large amount of missing transverse momentum attributed to the undetected neutrino. The rapidity distributions of the three charged leptons are shown in Figure 3.5, and the $p_{T}$ distributions for the leptons are shown in Figure 3.6. Since the $Z$ boson can be fully reconstructed from the charged lepton pair, we also show its $p_{T}$ distribution in Figure 3.7. These plots, generated in WHIZARD, 


\section{Backgrounds to WZ Scattering}

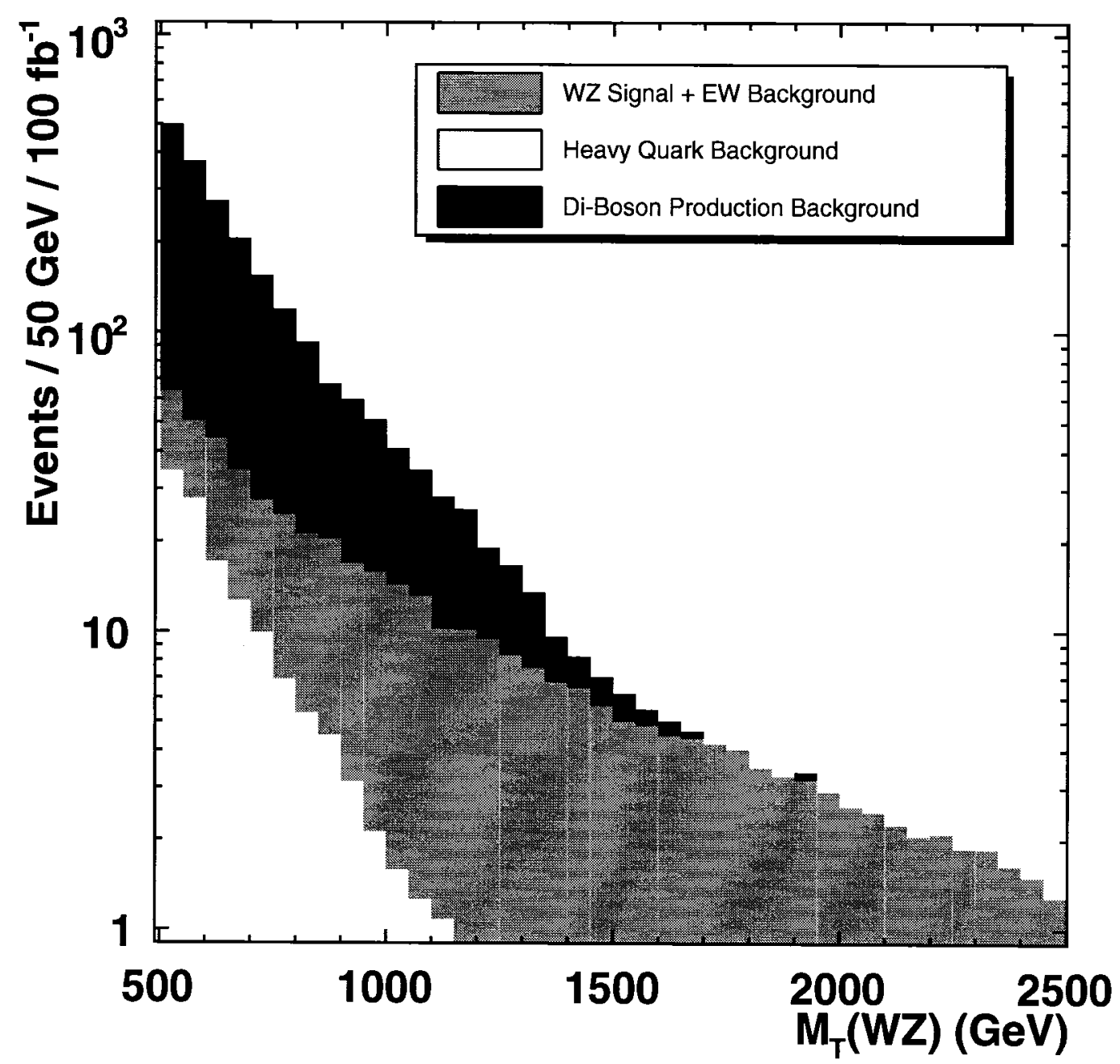

Figure 3.4: Transverse mass distributions of the backgrounds to $W Z$ scattering before imposing cuts for an integrated luminosity of $\mathcal{L}=100 \mathrm{fb}^{-1}$. 
show the distributions for both the resonant $W Z$ signal and for the electroweak background. Since the electroweak background is the most difficult background to reduce, we will choose our cuts in order to most effectively reduce this background while retaining as much of the signal as possible. The leptonic distributions for the heavy quark and di-boson production backgrounds are not shown for clarity; these backgrounds will be easily suppressed anyway, using the cuts derived from these plots.

The signal events have low jet activity in the central rapidity region of the detector. The two jets from the signal tend to appear in the forward/backward (large rapidity) region with energy on the order of $1 \mathrm{TeV}$ and $p_{T} \sim M_{W} / 2$. In order to enhance the signal to background ratio, we accept only those events in which the jets fit these general characteristics. This is referred to as a forward jet tag. Applying a forward jet tag is especially useful in suppressing the background of Figure 3.2 due to di-boson production, in which there are no final state quark jets. It is possible to tag two high $p_{T}$ forward jets, and although this significantly enhances the signal to background ratio, we will see that it proves to be costly for the signal events. Tagging a single energetic jet can sufficiently reduce the backgrounds, but can be far more efficient in retaining the signal. This result will be shown in Chapter 4.

The jets from the the electroweak and heavy quark backgrounds (Figures 3.1 and 3.3) are generally more central and have higher $p_{T}$ than the jets from the signal events. Therefore, if we reject events involving low rapidity, high $p_{T}$ jets, the electroweak and heavy quark backgrounds will be reduced. This is called a central jet veto. The rapidity, transverse momentum and energy distributions of the jets are shown in Figures 3.8 - 3.10 for the electroweak background and for the singly charged 
resonant signal.

Based on the kinematic distributions presented in this section, we choose the kinematic cuts shown in Table 3.1. Note that the central jet veto cuts are to be interpreted as: "Reject all events with jets having $|y|<2.0$ and $p_{T}>100 \mathrm{GeV}$." 


\begin{tabular}{|c|c|}
\hline Leptonic Cuts & Jet Cuts \\
\hline$|y(\ell)|<2.0$ & $2.0<\left|y\left(j_{\text {tag }}\right)\right|<5.0$ \\
\hline$p_{T}(\ell)>125 \mathrm{GeV}$ & $p_{T}\left(j_{\text {tag }}\right)>20 \mathrm{GeV}$ \\
\hline$p_{T}^{\text {miss }}>125 \mathrm{GeV}$ & $E\left(j_{\text {tag }}\right)>400 \mathrm{GeV}$ \\
\hline$p_{T}(Z)>250 \mathrm{GeV}$ & $\left|y\left(j_{\text {veto }}\right)\right|<2.0$ \\
\hline$M_{T}(\mathrm{WZ})>500 \mathrm{GeV}$ & $p_{T}\left(j_{\text {veto }}\right)>100 \mathrm{GeV}$ \\
\hline
\end{tabular}

Table 3.1: Leptonic cuts and jet cuts used in $W Z$ scattering to enhance the signal to background ratio. Note that the central jet veto cuts are to be interpreted as: "Reject all events with jets having $|y|<2.0$ and $p_{T}>100 \mathrm{GeV}$."
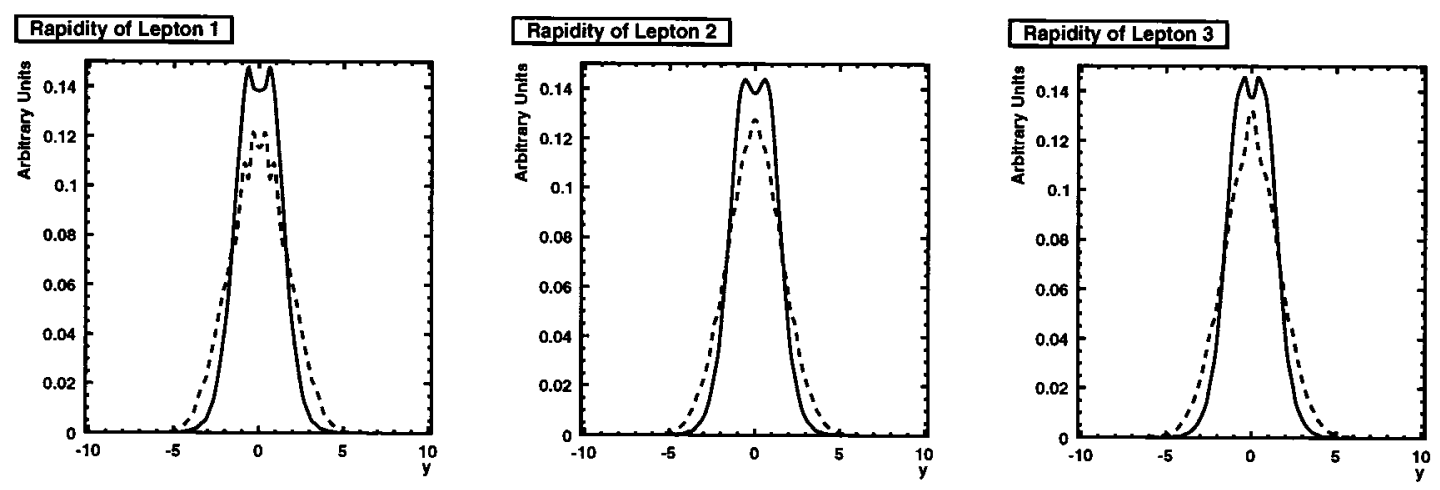

Figure 3.5: Rapidity distributions for the three charged leptons. The signal events are shown as the solid line, and the electroweak background events are shown as the dashed line. 

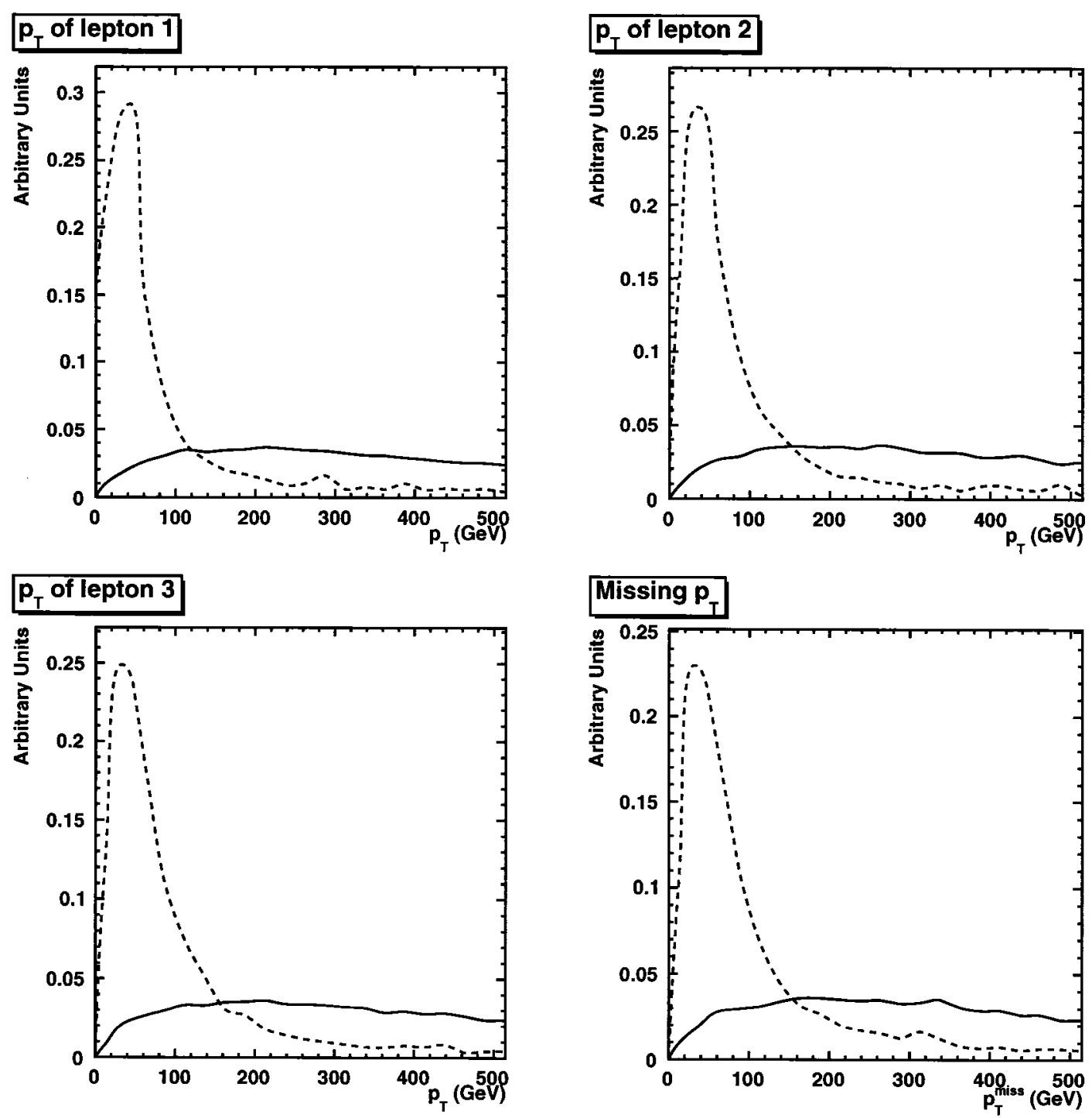

Figure 3.6: Transverse momentum distributions for the three charged leptons and for the missing transverse momentum. The signal events are shown as the solid line, and the electroweak background events are shown as the dashed line. 


\section{Z Transverse Momentum Distribution}

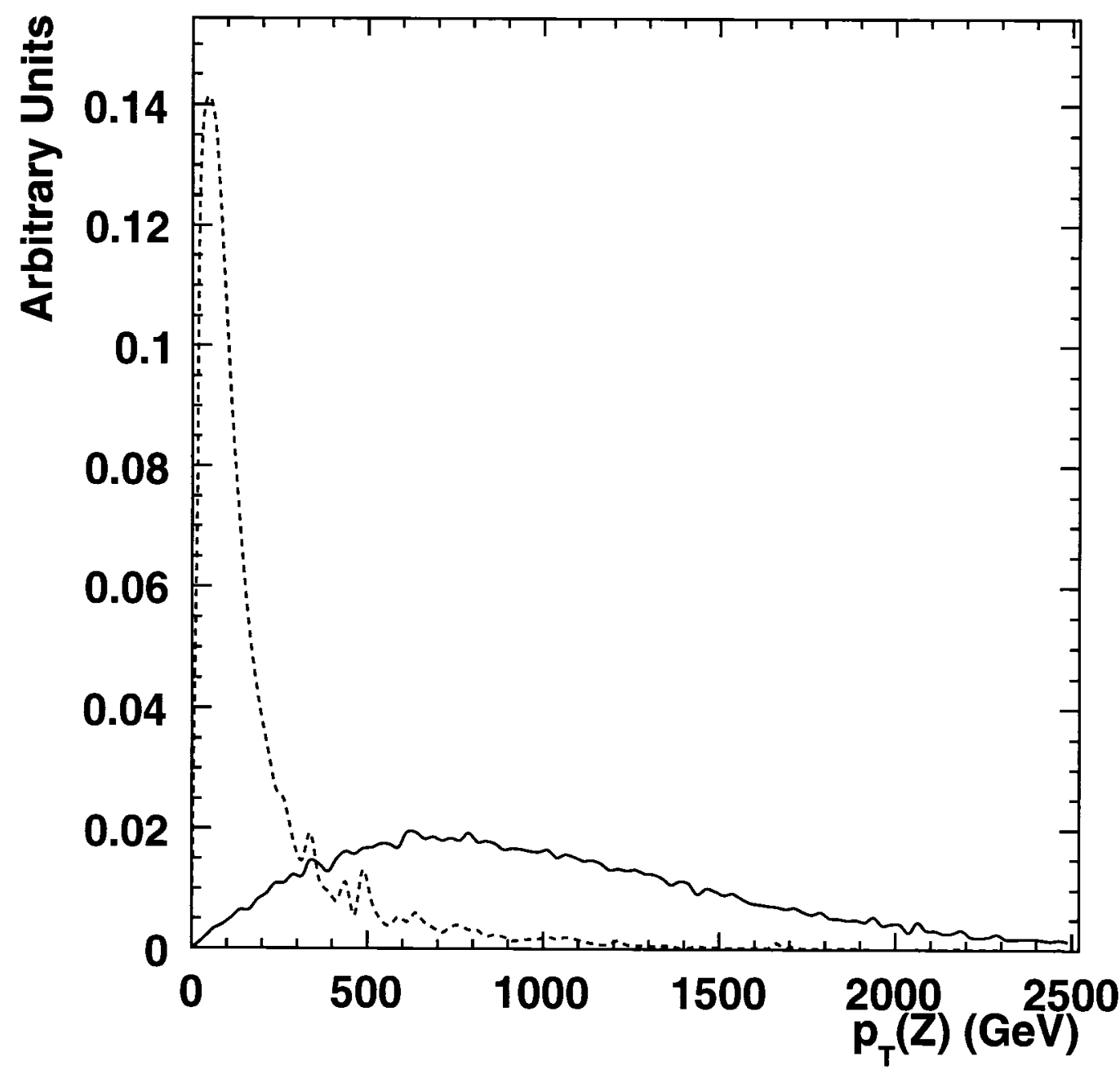

Figure 3.7: Transverse momentum distributions for the Z boson. The signal events are shown as the solid line, and the electroweak background events are shown as the dashed line. 


\section{Rapidity of jet 1}

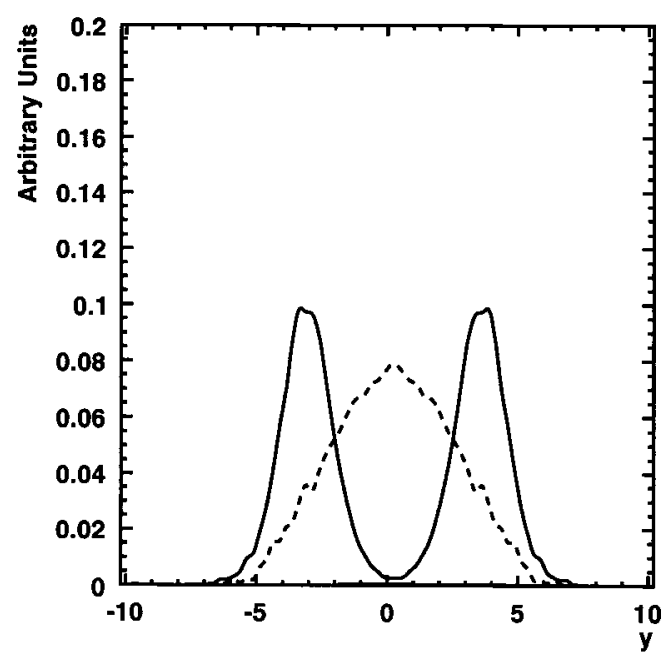

\section{Rapidity of jet 2}

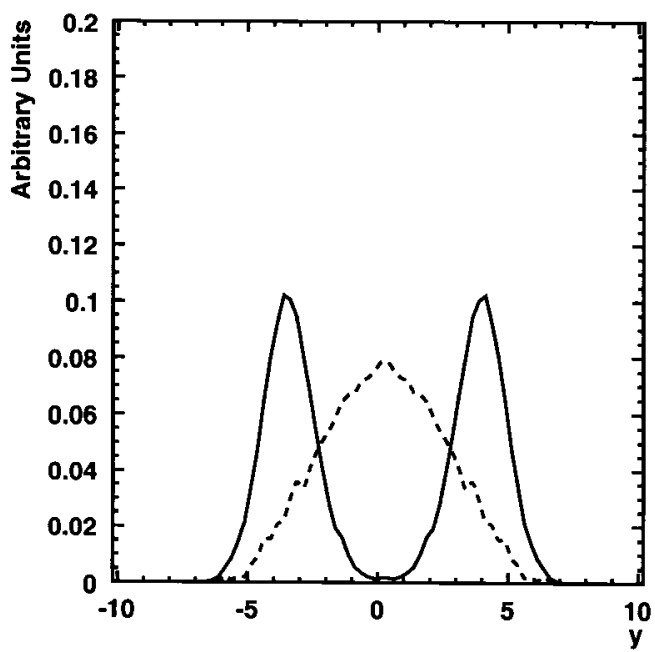

Figure 3.8: Rapidity distributions for the final state quark jets. The signal events are shown as the solid line, and the electroweak background events are shown as the dashed line.
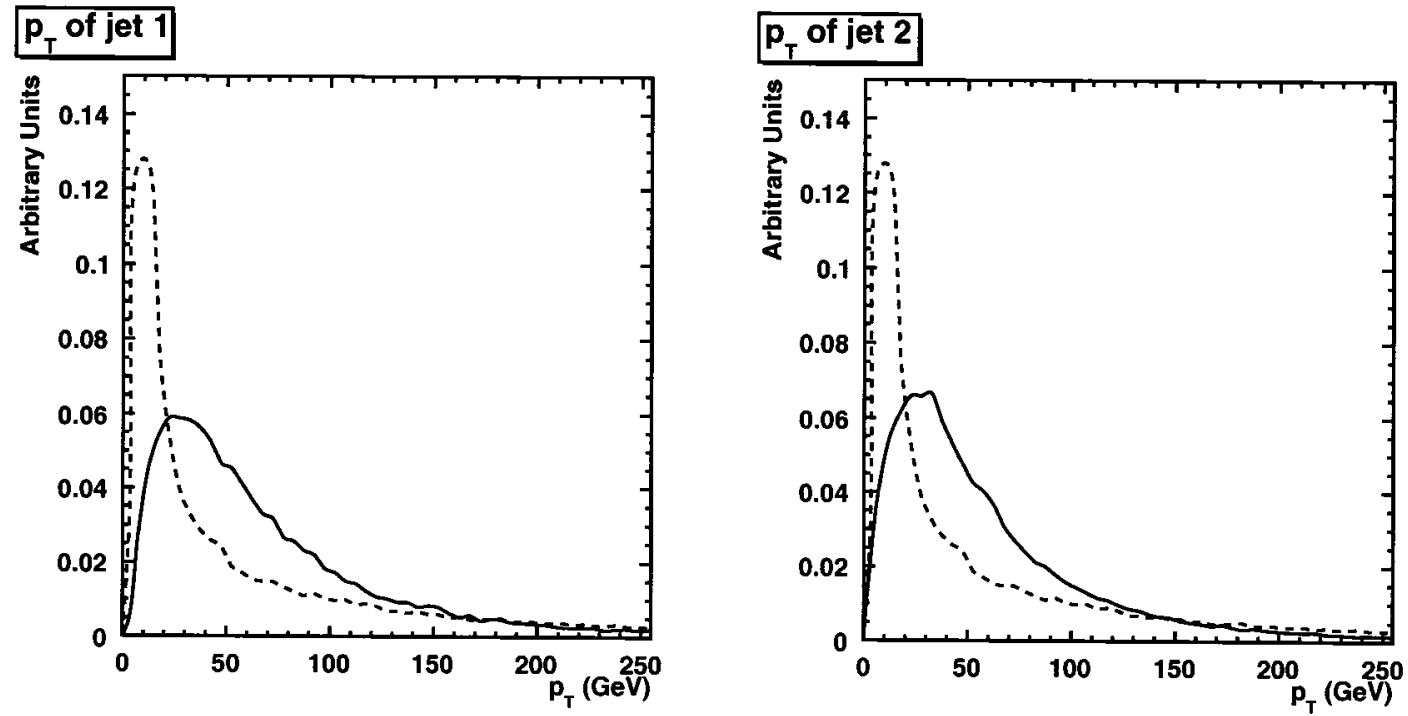

Figure 3.9: Transverse momentum distributions for the final state quark jets. The signal events are shown as the solid line, and the electroweak background events are shown as the dashed line. 

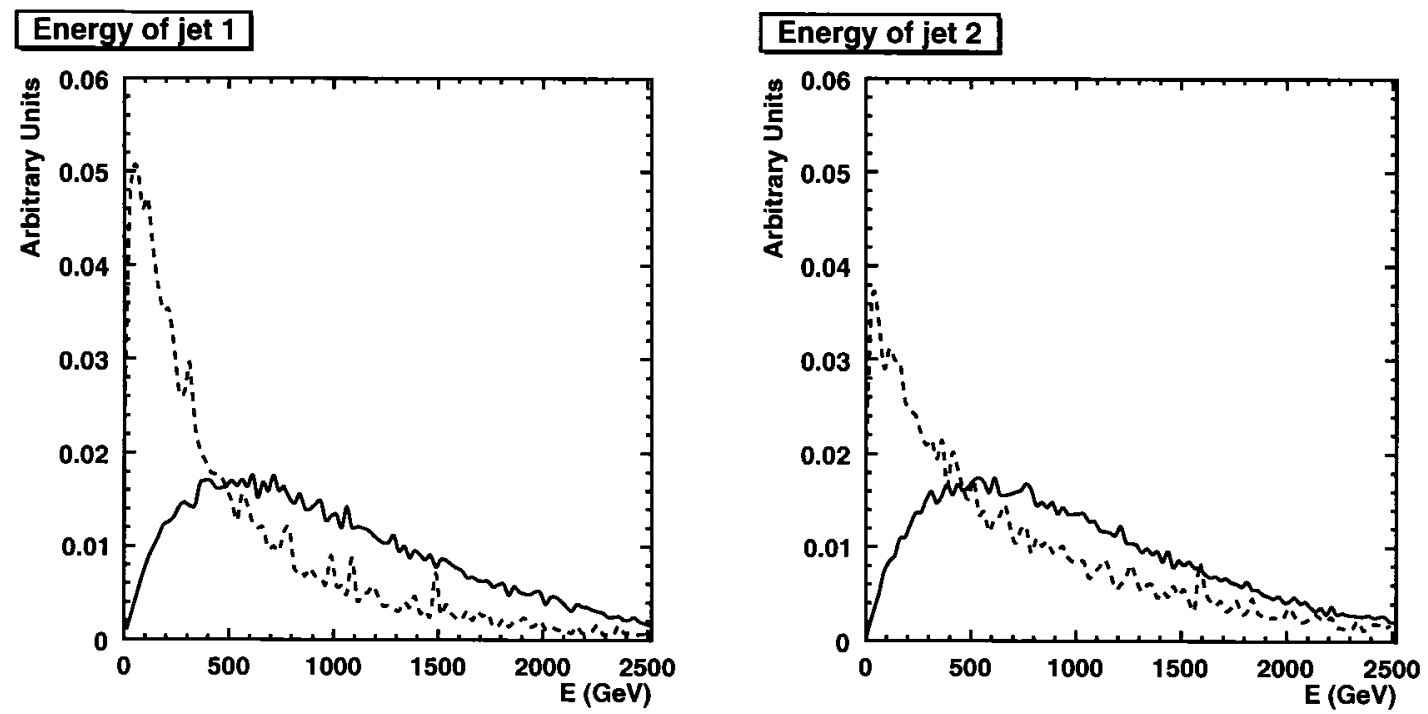

Figure 3.10: Energy distributions for the final state quark jets. The signal events are shown as the solid line, and the electroweak background events are shown as the dashed line. 


\section{Chapter 4}

\section{Results}

Figure 4.1 shows the transverse mass distribution for the $W Z$ signal in the Littlest Higgs model for an integrated luminosity of $\mathcal{L}=100 \mathrm{fb}^{-1}$, together with the background after the cuts of Table 3.1 have been applied. This plot was generated using WHIZARD with a Standard Model Higgs boson mass of $M_{H}=120 \mathrm{GeV}$, a heavy Higgs triplet mass of $M_{\Phi}=1.5 \mathrm{TeV}$, and a triplet vacuum expectation value of $v^{\prime} / v$ $=0.2$. We see that the singly-charged heavy Higgs signal can clearly be observed above the background remnant. However, this triplet vev of $v^{\prime} / v=0.2$ is four times larger than the theoretical upper limit $v^{\prime} / v<0.05$, given by equation (1.46). Since the resonant signal cross section is proportional to $\left(v^{\prime} / v\right)^{2}$, reducing the triplet vev by a factor of four reduces the resonant peak by a factor of 16 . If we were to plot the transverse mass distribution using $v^{\prime} / v=0.05$, the signal would not be observable above the background. Note that for the decay width, $\Gamma_{\Phi^{+}}$, we only included the $\Phi^{+} \rightarrow W^{+} Z$ decay mode. Including the $\Phi^{+} \rightarrow W^{+} H$ decay mode would decrease the 
$W Z$ scattering cross section by a factor of two, but would also lead to a second final state of comparable significance.

A similar plot using $M_{\Phi}=2 T e V$ is shown in Figure 4.2. Once again, the resonant signal can be observed above the background after cuts. However, this was also plotted using a triplet vev of $v^{\prime} / v=0.2$. If a more theoretically favoured value of $v^{\prime} / v=0.05$ were used, the signal would be reduced and would not be observable above the background. Figure 4.3 shows a similar plot using $M_{\Phi}=1 \mathrm{TeV}$. We notice that even with a triplet vev as large as $v^{\prime} / v=0.2$, the resonant signal is significantly smaller than in Figures 4.1 and 4.2 for triplet masses of $1.5 \mathrm{TeV}$ and $2 \mathrm{TeV}$.

The results shown in Figures 4.1 - 4.3 were calculated using the cuts given in Table 3.1, with a single forward jet tag. One can also investigate the effect of forward tagging both the jets. The effect of this double tagging is shown in Figure 4.4 for $M_{\Phi}=1.5 \mathrm{TeV}$. Comparing this with Figure 4.1, we see that, although the background has been reduced and the signal to background ratio has been improved, the signal itself has also been significantly reduced. Once again, this signal would be reduced even further if $v^{\prime} / v=0.05$ were used in the calculation. One should also note that Figures 4.1 - 4.4 show the transverse mass distribution for $W^{+} Z$ scattering; $W^{-} Z$ scattering can be included by multiplying the number of events in these figures by a factor of two. This would improve the number of signal events, but not enough to observe the signal if a triplet vev of $v^{\prime} / v=0.05$ were used. 


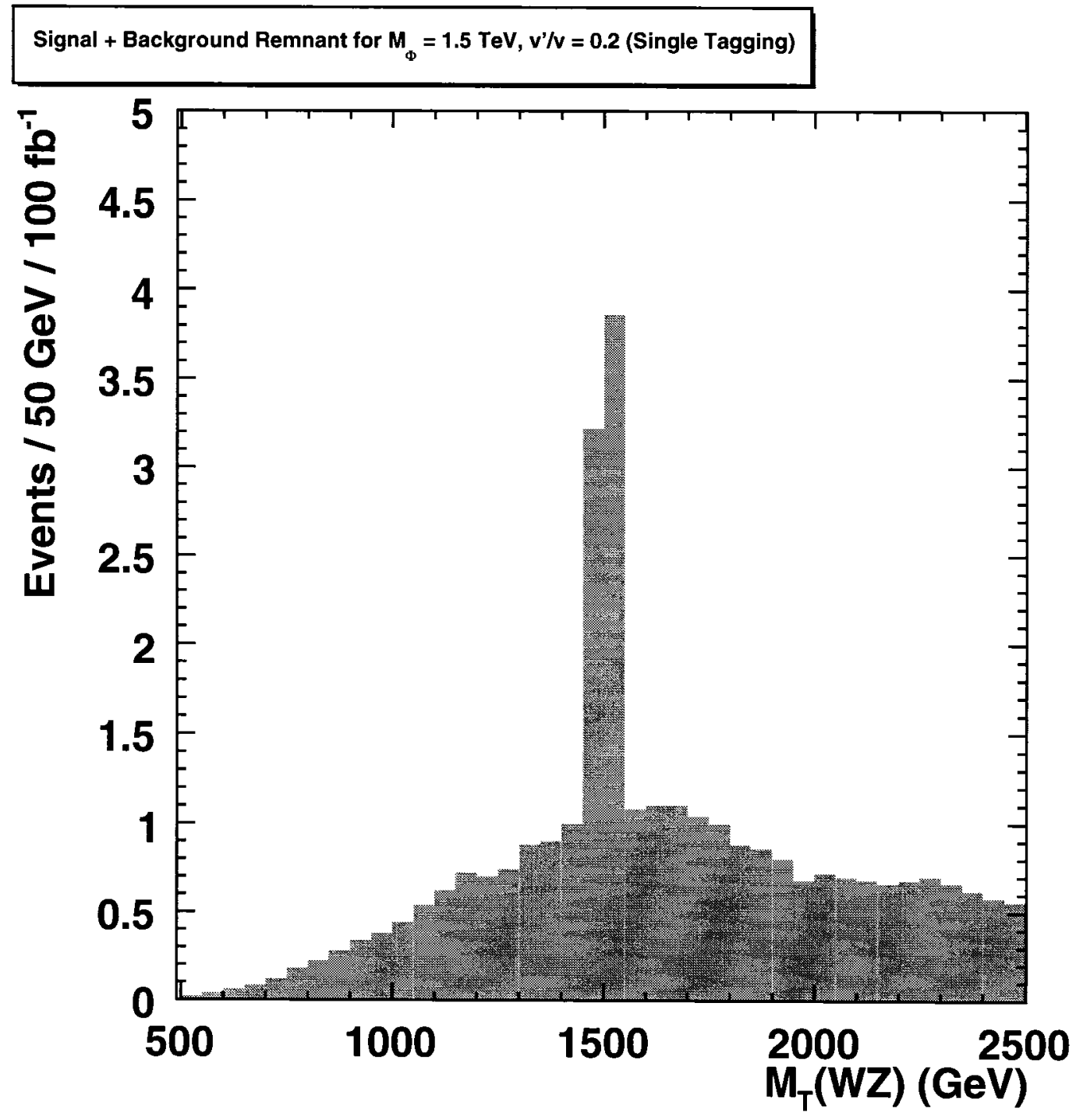

Figure 4.1: Transverse mass distribution for the $W Z$ signal in the Littlest Higgs model, together with the background remnant after the cuts of Table 3.1, for an integrated luminosity of $\mathcal{L}=100 \mathrm{fb}^{-1}$. This plot was generated using WHIZARD with a Standard Model Higgs mass of $M_{H}=120 \mathrm{GeV}$, a heavy Higgs triplet mass of $M_{\Phi}=1.5 \mathrm{TeV}$, and a triplet vacuum expectation value of $v^{\prime} / v=0.2$. 


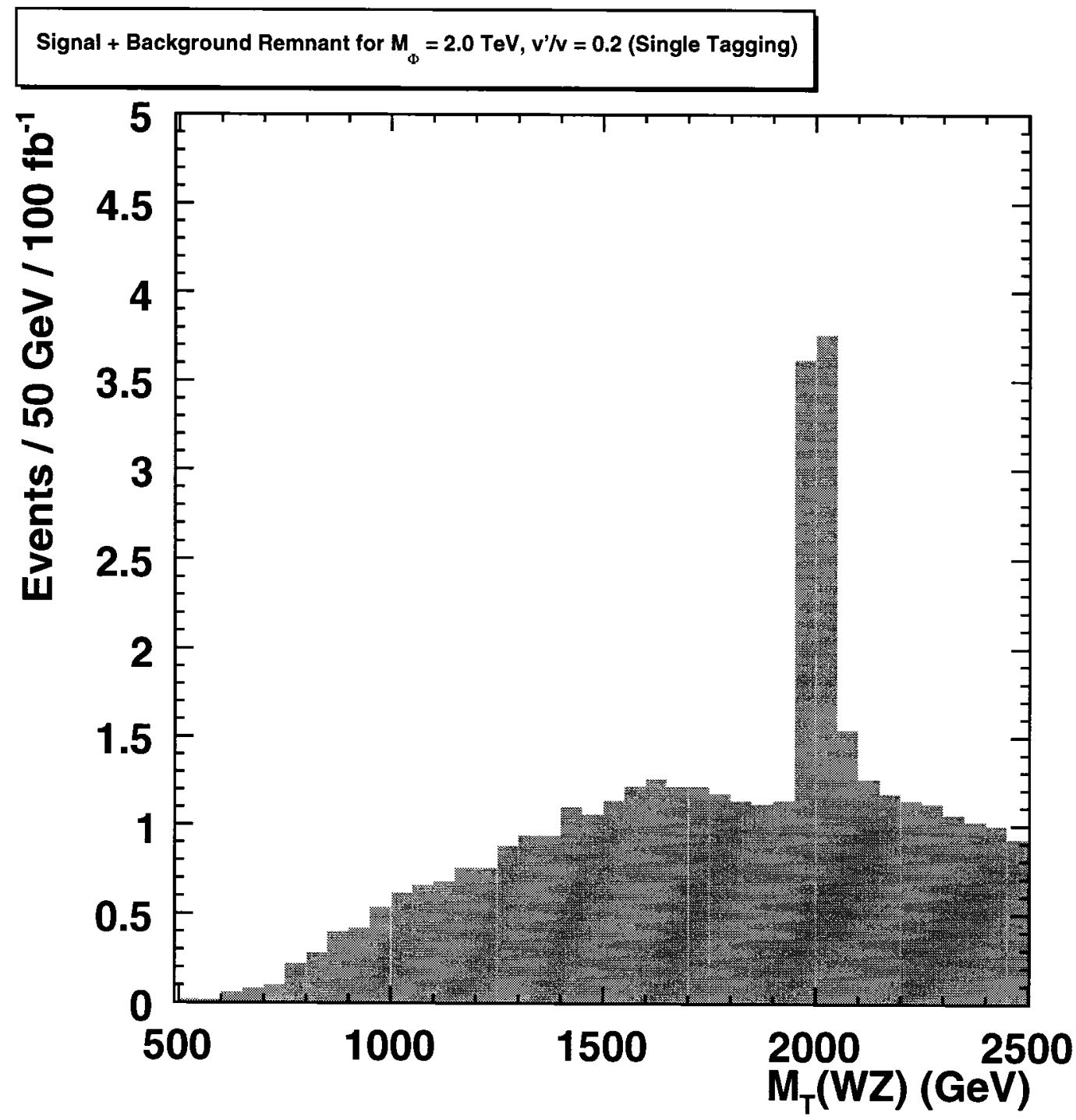

Figure 4.2: Transverse mass distribution for the $W Z$ signal in the Littlest Higgs model, together with the background remnant after the cuts of Table 3.1, for an integrated luminosity of $\mathcal{L}=100 \mathrm{fb}^{-1}$. This plot was generated using WHIZARD with a Standard Model Higgs mass of $M_{H}=120 \mathrm{GeV}$, a heavy Higgs triplet mass of $M_{\Phi}=2.0 \mathrm{TeV}$, and a triplet vacuum expectation value of $v^{\prime} / v=0.2$. 


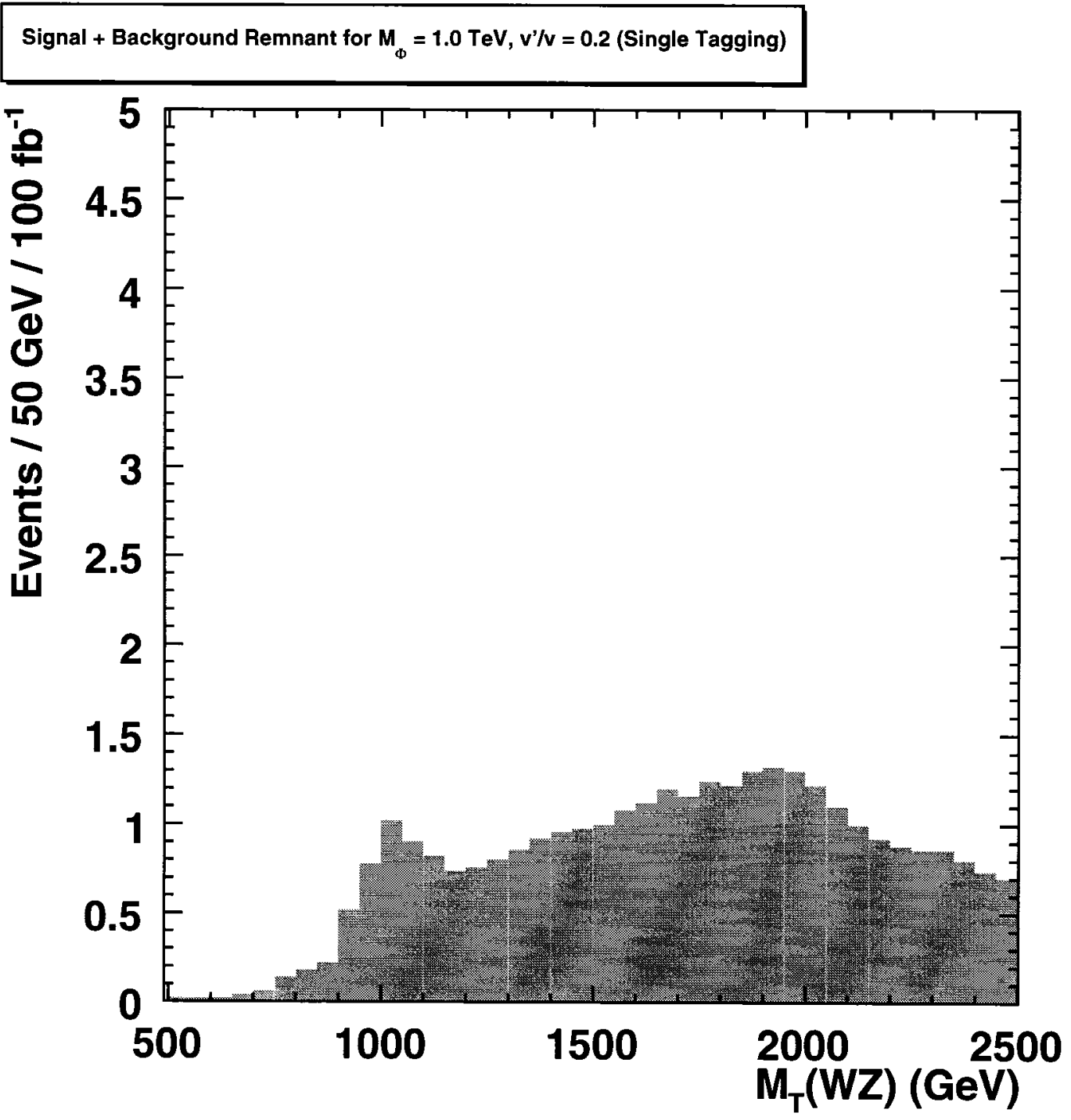

Figure 4.3: Transverse mass distribution for the $W Z$ signal in the Littlest Higgs model, together with the background remnant after the cuts of Table 3.1, for an integrated luminosity of $\mathcal{L}=100 \mathrm{fb}^{-1}$. This plot was generated using WHIZARD with a Standard Model Higgs mass of $M_{H}=120 \mathrm{GeV}$, a heavy Higgs triplet mass of $M_{\Phi}=1.0 \mathrm{TeV}$, and a triplet vacuum expectation value of $v^{\prime} / v=0.2$. 


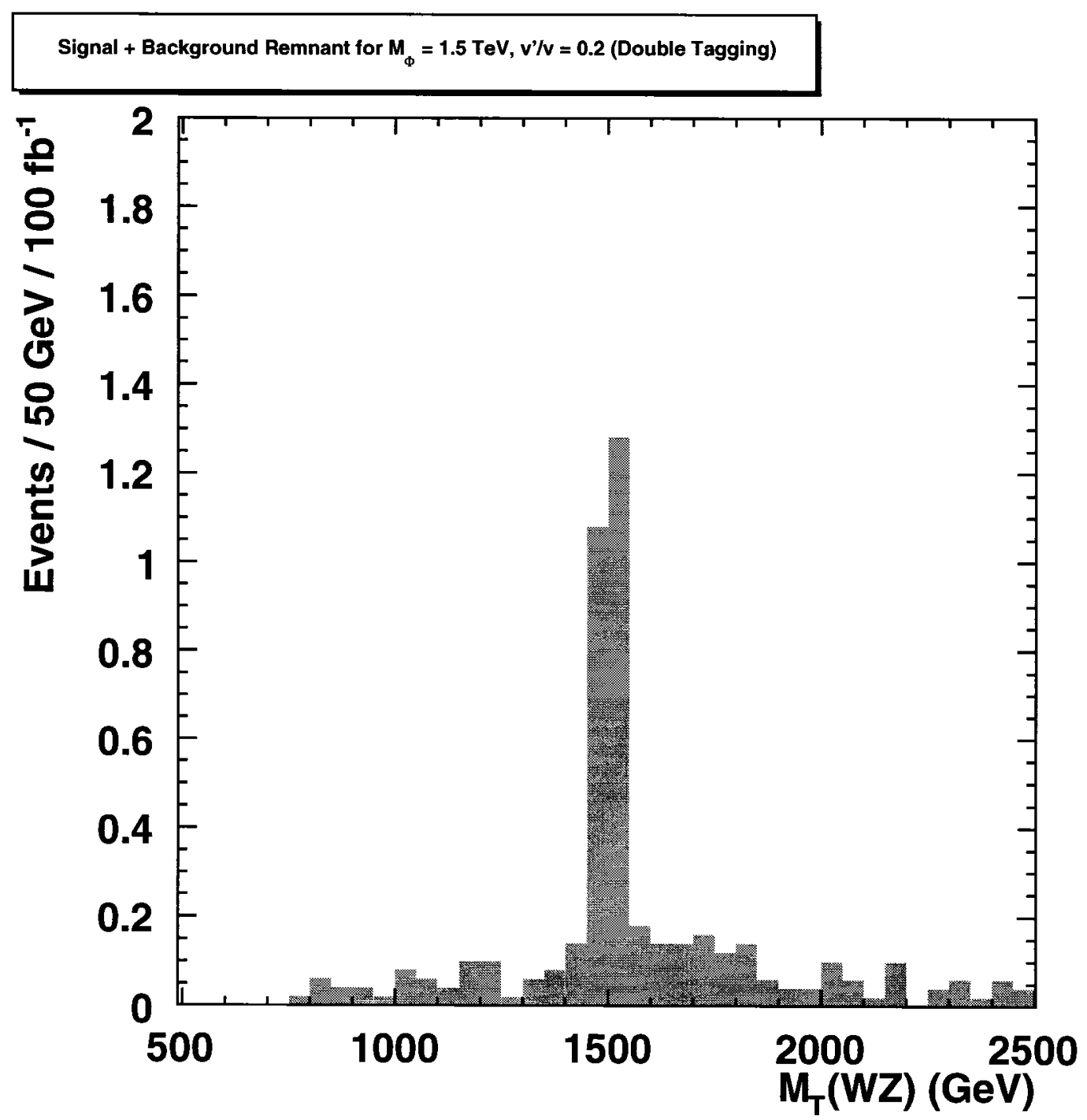

Figure 4.4: Transverse mass distribution for the $W Z$ signal in the Littlest Higgs model, together with the background remnant after the cuts of Table 3.1, for an integrated luminosity of $\mathcal{L}=100 \mathrm{fb}^{-1}$. This plot was generated using WHIZARD with a Standard Model Higgs mass of $M_{H}=120 \mathrm{GeV}$, a heavy Higgs triplet mass of $M_{\Phi}=1.5 \mathrm{TeV}$, and a triplet vacuum expectation value of $v^{\prime} / v=0.2$. Double forward jet tagging was used in this plot. 


\section{Chapter 5}

\section{Conclusions}

In this thesis, we investigated $W Z$ scattering at the LHC in the Littlest Higgs Model, which involves a singly-charged heavy Higgs resonance. The production of a singlycharged heavy Higgs boson through $W Z$ scattering does not occur in two Higgs doublet models such as Supersymmetry, so if this process were experimentally observed, it would provide evidence for the existence of a Higgs triplet.

The $W_{L} Z_{L}$ scattering signal cross section was computed in Chapter 2 using the Effective Vector Boson Approximation and also using the Goldstone Equivalence Theorem. This result is shown in Figure 2.5, using the parameters $M_{\Phi}=1.5 \mathrm{TeV}$, $M_{H}=120 \mathrm{GeV}$ and $v^{\prime} / v=0.05$. The calculation using longitudinally polarized gauge bosons agrees quite well with the calculation using the Goldstone Equivalence Theorem, in which the external gauge bosons are replaced by the corresponding Goldstone bosons. As expected, this agreement was found to be especially good at high invariant mass. 
Background processes to the $W Z$ signal were investigated in Chapter 3 to determine whether the signal could be experimentally observed at the LHC. Using the WHIZARD event generator, three types of background processes were considered: the electroweak background, the di-boson production background and the heavy quark background. Using appropriate kinematic cuts, shown in Table 3.1, the signal to background ratio was greatly improved. The signal and background remnant were plotted in Figures 4.1 - 4.3 using heavy Higgs triplet masses of $1 \mathrm{TeV}, 1.5 \mathrm{TeV}$, and $2 \mathrm{TeV}$, and by tagging a single energetic forward jet. We see that the signal is easily observed above the background remnant if a triplet vacuum expectation value of $v^{\prime} / v=0.2$ is used in the calculation. However, the signal would not be observable if a more theoretically likely value of $v^{\prime} / v=0.05$ were used.

The effect of forward tagging both jets was also examined for $M_{\Phi}=1.5 \mathrm{TeV}$. We found that while this double tagging reduced the background and improved the signal to background ratio, it proved to be costly to the signal. This can be seen by comparing Figures 4.1 and 4.4 .

Although the $W Z$ scattering channel does not appear to be very promising in the Littlest Higgs model, it would be instructive to determine whether this signal could be experimentally observed in other Higgs triplet models. For example, the Higgs triplet model of Georgi and Machacek predicts a higher triplet vacuum expectation value [37], and could provide an interesting signal for $W Z$ scattering. This would also allow us to distinguish between a variety of Higgs triplet models and, as data from the LHC become available, will allow us to determine which of these models, if any, are correct. 


\section{Appendix A}

\section{Feynman Rules}

In order to calculate the scattering amplitude for $W Z$ scattering, we need to know the Feynman rules relevant to this process. The Feynman rules for the relevant interaction vertices are shown in Table A.1. For a more extensive list of Feynman rules in the Littlest Higgs model, see [23].

The propagator for a scalar particle of mass $M$ and four-momentum $k_{\mu}$ is given by

$$
\frac{i}{k^{2}-M^{2}}
$$

The propagator for a vector boson of mass $M_{V}$ and four-momentum $k_{\mu}$ in the unitary gauge is given by

$$
\frac{i}{k^{2}-M_{V}^{2}}\left(-g_{\mu \nu}+\frac{k_{\mu} k_{\nu}}{M_{V}^{2}}\right)
$$

Scattering amplitudes are calculated by including factors for the appropriate interaction vertices and propagator, and for the case of external gauge bosons, the polariza- 


\begin{tabular}{|c|c|}
\hline Particles & Vertices \\
\hline$Z_{\mu} Z_{\nu} H$ & $\frac{i}{2} \frac{g^{2}}{c_{W}^{2}} v g_{\mu \nu}\left(1-\frac{v^{2}}{3 f^{2}}-\frac{1}{2} s_{0}^{2}+4 \sqrt{2} s_{0} \frac{v^{\prime}}{v}\right.$ \\
& $\left.-\frac{1}{2}\left(\left(c^{2}-s^{2}\right)^{2}+5\left(c^{2}-s^{\prime 2}\right)^{2}\right) \frac{v^{2}}{f^{2}}\right)$ \\
\hline$Z_{\mu} Z_{\nu} \Phi^{0}$ & $-\frac{i}{2} \frac{g^{2}}{c_{W}^{2}}\left(v s_{0}-4 \sqrt{2} v^{\prime}\right) g_{\mu \nu}$ \\
\hline$W_{\mu}^{+} W_{\nu}^{-} H$ & $\frac{i}{2} g^{2} v g_{\mu \nu}\left(1-\frac{v^{2}}{3 f^{2}}-\frac{1}{2}\left(c^{2}-s^{2}\right)^{2} \frac{v^{2}}{f^{2}}-\frac{1}{2} s_{0}^{2}-2 \sqrt{2} s_{0} \frac{v^{\prime}}{v}\right)$ \\
\hline$W_{\mu}^{+} W_{\nu}^{-} \Phi^{0}$ & $-\frac{i}{2} g^{2}\left(s_{0} v-2 \sqrt{2} v^{\prime}\right) g_{\mu \nu}$ \\
\hline$W_{\mu}^{+} Z_{\nu} \Phi^{-}$ & $-i \frac{g^{2}}{c_{W}} v^{\prime} g_{\mu \nu}$ \\
\hline$Z_{\mu} Z_{\nu} W_{\rho}^{+} W_{\sigma}^{-}$ & $-i g^{2} c_{W}^{2}\left(2 g_{\mu \nu} g_{\rho \sigma}-g_{\mu \rho} g_{\nu \sigma}-g_{\nu \rho} g_{\mu \sigma}\right)$ \\
\hline$W_{\mu}^{+}\left(k_{1}\right) W_{\nu}^{-}\left(k_{2}\right) Z_{\rho}\left(k_{3}\right)$ & $i g c_{W}\left[g_{\mu \nu}\left(k_{1}-k_{2}\right)_{\rho}+g_{\nu \rho}\left(k_{2}-k_{3}\right)_{\mu}+g_{\rho \mu}\left(k_{3}-k_{1}\right)_{\nu}\right]$ \\
\hline
\end{tabular}

Table A.1: Feynman rules required to calculate the parton level amplitude for $W Z$ scattering in the Littlest Higgs model. All particles are assumed to be outgoing.

tion vectors $\epsilon_{\mu}$ are also included.

We also wish to use the Goldstone Equivalence Theorem to calculate the parton-level $W_{L} Z_{L}$ scattering amplitude. In order to do this, we replace the external longitudinal gauge bosons with the corresponding unphysical Goldstone bosons. The Feynman diagrams for this process are shown in Figure 2.4. In order to calculate the scattering amplitude for these diagrams, we need to derive the scalar Feynman rules involving Goldstone bosons in the Littlest Higgs model.

We begin with the Coleman-Weinberg potential of the Littlest Higgs model

$$
V=\lambda_{\phi^{2}} f^{2} \operatorname{Tr}\left(\phi^{\dagger} \phi\right)+i \lambda_{h \phi h} f\left(h \phi^{\dagger} h^{T}-h^{*} \phi h^{\dagger}\right)-\mu^{2} h h^{\dagger}+\lambda_{h^{4}}\left(h h^{\dagger}\right)^{2}
$$


where the Higgs doublet and triplet are given by

$$
h=\left(h^{+}, h^{0}\right), \quad \phi=\left(\begin{array}{cc}
\phi^{++} & \frac{\phi^{+}}{\sqrt{2}} \\
\frac{\phi^{+}}{\sqrt{2}} & \phi^{0}
\end{array}\right) .
$$

The gauge eigenstates of the Higgs fields, $h$ and $\phi$, can be expressed in terms of the mass eigenstates:

$$
\begin{aligned}
h^{0} & =\left(c_{0} H-s_{0} \Phi^{0}+v\right) / \sqrt{2}+i\left(c_{P} G^{0}-s_{P} \Phi^{P}\right) / \sqrt{2} \\
\phi^{0} & =\left(s_{P} G^{0}+c_{P} \Phi^{P}\right) / \sqrt{2}-i\left(s_{0} H+c_{0} \Phi^{0}+\sqrt{2} v^{\prime}\right) / \sqrt{2} \\
h^{+} & =c_{+} G^{+}-s_{+} \Phi^{+} \\
\phi^{+} & =\left(s_{+} G^{+}+c_{+} \Phi^{+}\right) / i \\
\phi^{++} & =\Phi^{++} / i .
\end{aligned}
$$

The mass eigenstates are defined as follows: $H$ is a neutral scalar identified with the Standard Model Higgs boson, $\Phi^{0}, \Phi^{+}$and $\Phi^{++}$are the scalars of the heavy Higgs triplet, $\Phi^{P}$ is a neutral pseudoscalar, and $G^{+}$and $G^{0}$ are the Goldstone bosons that are eaten by the $W$ and $Z$ gauge bosons, giving them mass.

The mixing angles in equation (A.5) can be expessed in terms of the vacuum expectation values, $v$ and $v^{\prime}$ :

$$
\begin{aligned}
& s_{0} \simeq 2 \sqrt{2} \frac{v^{\prime}}{v}, \quad c_{0} \simeq 1-4 \frac{v^{\prime 2}}{v^{2}} \\
& s_{P}=\frac{2 \sqrt{2} v^{\prime}}{\sqrt{v^{2}+8 v^{\prime 2}}} \simeq 2 \sqrt{2} \frac{v^{\prime}}{v}, \quad c_{P}=\frac{v}{\sqrt{v^{2}+8 v^{\prime 2}}} \simeq 1-4 \frac{v^{\prime 2}}{v^{2}} \\
& s_{+}=\frac{2 v^{\prime}}{\sqrt{v^{2}+4 v^{\prime 2}}} \simeq 2 \frac{v^{\prime}}{v}, \quad c_{+}=\frac{v}{\sqrt{v^{2}+4 v^{\prime 2}}} \simeq 1-2 \frac{v^{\prime 2}}{v^{2}}
\end{aligned}
$$


We see that to leading order, $s_{0}=s_{P}=\sqrt{2} s_{+}$.

The couplings, $\lambda_{\phi^{2}}, \lambda_{h \phi h}$, and $\lambda_{h^{4}}$, in the Coleman-Weinberg potential can also be expressed to leading order as

$$
\begin{aligned}
\lambda_{\phi^{2}} & \simeq \frac{M_{\Phi}^{2}}{f^{2}} \\
\lambda_{h \phi h} & \simeq \frac{2 M_{\Phi}^{2} v^{\prime}}{v^{2} f} \\
\lambda_{h^{4}} & =\frac{1}{4} \lambda_{\phi^{2}} \simeq \frac{M_{\Phi}^{2}}{4 f^{2}}
\end{aligned}
$$

It is now straightforward to calculate the scalar Feynman rules from the Coleman-Weinberg potential. We simply substitute equations (A.4) and (A.5) into the Coleman-Weinberg potential of equation (A.3) and extract the coefficients for each term. We adopt the convention that the Feynman rule is equal to $i \mathcal{L}$. For example, the Feynman rule for the $G^{0} G^{0} H$ vertex is the coefficient of the resulting $G^{0} G^{0} H$ term, multiplied by $i$. The scalar Feynman rules for the relevant vertices are

$$
\begin{aligned}
G^{0} G^{0} H & =i \lambda_{h \phi h} f\left(\frac{1}{\sqrt{2}} s_{0} c_{P}^{2}-\sqrt{2} c_{0} s_{P} c_{P}\right)+i \lambda_{h^{4}} v c_{0} c_{P}^{2} \\
G^{0} G^{0} \Phi^{0} & =i \lambda_{h \phi h} f\left(\frac{1}{\sqrt{2}} c_{0} c_{P}^{2}+\sqrt{2} s_{0} s_{P} c_{P}\right)-i \lambda_{h^{4}} v s_{0} c_{P}^{2} \\
G^{+} G^{-} H & =-2 i \lambda_{h \phi h} f c_{0} s_{+} c_{+}+2 i \lambda_{h^{4}} v c_{0} c_{+}^{2} \\
G^{+} G^{-} \Phi^{0} & =2 i \lambda_{h \phi h} f s_{0} s_{+} c_{+}-2 i \lambda_{h^{4}} v s_{0} c_{+}^{2} \\
G^{+} G^{0} \Phi^{-} & =\lambda_{h \phi h} f c_{P} \\
G^{0} G^{0} G^{+} G^{-} & =i \lambda_{h^{4}} c_{+}^{2} c_{P}^{2} .
\end{aligned}
$$


Substituting equations (A.6) and (A.7) into equation (A.8) allows us to express these Feynman rules in terms of the physical parameters, $f, M_{\Phi}, v$ and $v^{\prime}$ of the Littlest Higgs model. The results are shown in Table A.2 along with the Feynman rule for the $G^{+} G^{0} W^{-}$vertex [23], which is needed to calculate the amplitude for diagrams (c) and (d) of Figure 2.4.

\begin{tabular}{|c|c|}
\hline Particles & Vertices \\
\hline$G^{0} G^{0} H$ & $2 i \frac{M_{\Phi}^{2}}{v}\left[\frac{v^{2}}{f^{2}}\left(\frac{1}{4}-3 \frac{v^{\prime 2}}{v^{2}}\right)-4 \frac{v^{\prime 2}}{v^{2}}\right]$ \\
\hline$G^{0} G^{0} \Phi^{0}$ & $2 \sqrt{2} i \frac{M_{\Phi}^{2}}{v}\left[\left(1-\frac{v^{2}}{2 f^{2}}\right) \frac{v^{\prime}}{v}+4 \frac{v^{3}}{v^{3}}\right]$ \\
\hline$G^{+} G^{-} H$ & $i \frac{M_{\Phi}^{2}}{v}\left[\frac{v^{2}}{f^{2}}\left(\frac{1}{2}-4 \frac{v^{\prime 2}}{v^{2}}\right)-8 \frac{v^{\prime 2}}{v^{2}}\right]$ \\
\hline$G^{+} G^{-} \Phi^{0}$ & $-\sqrt{2} i \frac{M_{\Phi}^{2} v^{\prime}}{v^{2}}\left(\frac{v^{2}}{f^{2}}-16 \frac{v^{2}}{v^{2}}\right)$ \\
\hline$G^{+} G^{0} \Phi^{-}$ & $2 \frac{M_{\Phi}^{2} v^{\prime}}{v^{2}}\left(1-4 \frac{v^{2}}{v^{2}}\right)$ \\
\hline$G^{0} G^{0} G^{+} G^{-}$ & $i \frac{M_{\Phi}^{2}}{2 f^{2}}\left(1-12 \frac{v^{2}}{v^{2}}\right)$ \\
\hline$W_{\mu}^{+} G^{0}\left(k_{1}\right) G^{-}\left(k_{2}\right)$ & $-i \frac{g}{\sqrt{2}}\left(k_{1}-k_{2}\right)_{\mu}$ \\
\hline
\end{tabular}

Table A.2: Feynman rules required to calculate the parton level amplitude for $W_{L} Z_{L}$ scattering in the Littlest Higgs model using the Goldstone Equivalence Theorem. All particles are assumed to be outgoing. 


\section{References}

[1] S.L. Glashow. Partial Symmetries of Weak Interactions. Nucl. Phys., 22:579 $588,1961$.

[2] S. Weinberg. A Model of Leptons. Phys. Rev. Lett., 19:1264 - 1266, 1967.

[3] A. Salam. Elementary Particle Theory. Almqvist and Wiksells, Stockholm, 1969.

[4] H. Fritzch, M. Gell-Mann, and H. Leutwyler. Phys. Lett. B, 47:365, 1973.

[5] D. J. Gross and F. Wilczek. Asymptotically Free Gauge Theories. I. Phys. Rev. $D, 8: 3633-3652,1973$.

[6] S. Dawson. Introduction to Electroweak Symmetry Breaking. arXiv.org/hepph/9901280, 1999.

[7] W.-M. Yao et al. Review of Particle Physics. Journal of Physics G, 33:1, 2006.

[8] E. Fermi. Atti. Soc. It. Prog. Scient., 2:7 - 14, 1933.

[9] R. Chivukula and E. Simmons. Triviality and the Precision Bound on the Higgs Mass. Phys. Lett. B, 388:788, 1996. 
[10] N. Cabibbo, L. Maiani, G. Parisi, and R. Petronzio. Nucl. Phys. B, 158:295, 1979.

[11] S. Weinberg. Mass of the Higgs Boson. Phys. Rev. Lett., 36:294 - 296, 1976.

[12] M. Quiros. Perspectives in Higgs Physics. World Scientific, Singapore, 1997.

[13] LEP Electroweak Working Group. http://lepewwg.web.cern.ch/LEPEWWG/. Technical report.

[14] C. Quigg. Electroweak Symmetry Breaking and the Higgs Sector. Acta Phys. Polon. B, 30:2145 - 2192, 1999.

[15] S. Dawson. Electroweak Symmetry Breaking circa 2005. Int. J. Mod. Phys. A, $21: 1629-1641,2006$.

[16] G. Giudice. Theoretical Predictions for Collider Searches. Int. J. Mod. Phys. A, $19: 835-850,2004$.

[17] N. Arkani-Hamed, A.G. Cohen, and H. Georgi. Electroweak symmetry breaking from dimensional deconstruction. Phys. Lett. B, 513:232, 2001.

[18] M. Schmaltz and D. Tucker-Smith. Little Higgs Review. Ann. Rev. Nucl. Part. Sci., 55:229 - 270, 2005.

[19] T. Han, H.E. Logan, and L.-T. Wang. Smoking gun signatures of little Higgs models. JHEP, 0601:099, 2006.

[20] M. Schmaltz. The Simplest Little Higgs. JHEP, 0408:056, 2004. 
[21] N. Arkani-Hamed, A.G. Cohen, E. Katz, and A.E. Nelson. The Littlest Higgs. JHEP, 0207:034, 2002.

[22] H.-C. Cheng and I. Low. TeV Symmetry and the Little Hierarchy Problem. JHEP, 0309:051, 2003.

[23] T. Han, H.E. Logan, B. McElrath, and L.-T. Wang. Phenomenology of the Little Higgs Model. Phys. Rev. D, 67:095004, 2003.

[24] S.R. Coleman and E. Weinberg. Radiative Corrections as the Origin of Spontaneous Symmetry Breaking. Phys. Rev. D, 7:1888 - 1910, 1973.

[25] E. Asakawa, S. Kanemura, and J. Kanzaki. Potential for measuring the $H^{ \pm} W^{\mp} Z^{0}$ vertex from WZ fusion at the Large Hadron Collider. arXiv.org/hep-ph/0612271, 2007.

[26] M.S. Chanowitz and M.K. Gaillard. The TeV Physics of Strongly Interacting W's and Z's. Nucl. Phys. B, 261:379-431, 1985.

[27] S. Dawson. The Effective W Approximation. Nucl. Phys. B, 249:42 - 60, 1985.

[28] J. Pumplin, D.R. Stump, J. Huston, H.L Lai, P. Nadolsky, and W.K. Tung. New Generation of Parton Distributions with Uncertainties from Global QCD Analysis. JHEP, 0207:012, 2002.

[29] J. Cornwall, D. Levin, and G. Tiktopoulos. Derivation of gauge invariance from high-energy unitarity bounds on the S matrix. Phys. Rev. D, 10:1145 - 1167, 1974. 
[30] W. Kilian. WHIZARD 1.50: A generic Monte-Carlo integration and event generation package for multi-particle processes. LC-TOOL-2001-039.

[31] V. Barger, K. Cheung, T. Han, and R.J.N. Phillips. Strong $W^{+} W^{+}$scattering signals at pp supercolliders. Phys. Rev. D, 42:3052 - 3077, 1990.

[32] J. Bagger, V. Barger, K. Cheung, J. Gunion, T. Han, G.A. Ladinsky, R. Rosenfeld, and C.-P. Yuan. Strongly interacting WW system: Gold-plated modes. Phys. Rev. D, 49:1246 - 1264, 1994.

[33] J. Bagger, V. Barger, K. Cheung, J. Gunion, T. Han, G.A. Ladinsky, R. Rosenfeld, and C.-P. Yuan. CERN LHC Analysis of the strongly interacting $W W$ system: Gold-plated modes. Phys. Rev. D, 52:3878 - 3889, 1995.

[34] A. Pukhov, E. Boos, M. Dubinin, V. Edneral, V. Ilyin, D. Kovalenko, A. Kryukov, V. Savrin, S. Shichanin, and A. Semenov. CompHEP - a package for evaluation of Feynman diagrams and integration over multi-particle phase space. Preprint INP MSU 98-41/542, arXiv.org/hep-ph/9908288.

[35] T. Stelzer and W.F. Long. Automatic Generation of Tree Level Helicity Amplitudes. Comput. Phys. Commun., 81:357 - 371, 1994.

[36] M. Moretti, T. Ohl, and J Reuter. O’Mega: An Optimizing Matrix Element Generator. $L C-T O O L-2001-040$.

[37] H. Georgi and M. Machacek. Doubly Charged Higgs Bosons. Nucl. Phys. B, 262:463, 1985. 